\title{
Dentistry after Omicron variant: Introducing a New Biosafety Protocol in Prevention of COVID-19 in Dental Care
}

\author{
Andrej Thurzo ${ }^{1 *}$, Alexandra Bražinová ${ }^{2}$, Neda Markovská ${ }^{3}$, Iveta Waczulíková ${ }^{4}$, Martin Smatana ${ }^{5}$, Norbert Mo- \\ ravanský ${ }^{6}$, Wanda Urbanová ${ }^{7}$, Renáta Urban ${ }^{8}$, Veronika Kurilová ${ }^{9}$, Peter Sabaka ${ }^{10}$, Bela Mriňáková ${ }^{11}$, Ivan \\ Varga ${ }^{12}$, Ladislav Czakó ${ }^{13}$, Pavol Vitovič ${ }^{14,15}$, Helena Kosnáčová ${ }^{14,15}$, Jana Kaiferová ${ }^{8}$, Martin Strunga ${ }^{1}$, Branislav \\ Gális ${ }^{13}$, Georgia Fountoulaki ${ }^{3}$ and Bohuslav Novák ${ }^{1}$
}

1 Department of Stomatology and Maxillofacial Surgery, Faculty of Medicine, Comenius University in Bratislava, 81250 Bratislava, Slovakia; Thurzo3@uniba.sk, bohuslav.novak@fmed.uniba.sk, martin@3Dent.sk

2 Institute of Epidemiology, Faculty of Medicine, Comenius University in Bratislava, 81250 Bratislava, Slovakia; alexandra.brazinova@gmail.com

3 Department of Stomatology, Faculty of Medicine, Slovak Medical University in Bratislava, 83303 Slovakia; neda.markovska@gmail.com, tzina.fountoulaki@gmail.com

4 Department of Nuclear Physics and Biophysics, Faculty of Mathematics, Physics and Informatics, Comenius University, Mlynska dolina F1, 84248 Bratislava, Slovakia; iveta.waczulikova@fmph.uniba.sk

5 Ministry of Education, Science, Research and Sports of Slovak Republic, Slovakia; mar.smatana@gmail.com

6 Institute of Forensic Medicine, Faculty of Medicine Comenius University in Bratislava, Sasinkova 4, 81108 Bratislava, Slovakia; norbert.moravansky@forensic.help

7 Department of Orthodontics and Cleft Anomalies, Dental Clinic $3^{\text {rd }}$ Medical Faculty Charles University, Faculty Hospital Kralovske Vinohrady, Prague, Czech Republic; wanda.urbanova@gmail.com

$81^{\text {st }}$ Department of Dentistry, Faculty of Medicine Pavol Jozef Safarik University, Trieda SNP 1, 04011 Kosice, Slovakia; renyurban@gmail.com, kaiferova@gmail.com

9 Faculty of Electrical Engineering and Information Technology, Slovak University of Technology, Ilkovicova 3, 81219 Bratislava, Slovakia; veronika.hanuskova@gmail.com

10 Department of Infectiology and Geographical Medicine, Faculty of Medicine, Comenius University in Bratislava, Slovakia; petersabaka@gmail.com

$111^{\text {st }}$ Department of Oncology, Medical Faculty of Comenius University and St. Elisabeth Cancer Institute, 81250 Bratislava, Slovakia; bela.mrinakova@ousa.sk

12 Institute of Histology and Embryology, Medical Faculty of Comenius University in Bratislava, 81372 Bratislava, Slovakia; ivan.varga@fmed.uniba.sk

${ }_{13}$ Department of Oral and Maxillofacial Surgery of Medical Faculty of Comenius University and University Hospital Bratislava, Bratislava, Slovakia; czako@ionline.sk, brano.galis@gmail.com

14 Department of Genetics, Cancer Research Institute, Biomedical Research Center, Slovak Academy of Sciences, Dubravska Cesta 9, 84505 Bratislava, Slovakia; pavol.vitovic@fmed.uniba.sk

15 Department of Simulation and Virtual Medical Education, Faculty of Medicine, Comenius University in Bratislava, Sasinkova 4, 81272 Slovakia; helena.svobodova@fmed.uniba.sk

* Correspondence: Andrej.Thurzo@fmed.uniba.sk; Tel.: (+421 903110 107)

\begin{abstract}
Omicron variant is more invasive against immunity, but it's not more invasive in any change in degree against masks, and indoor air; than previous variants. With its arrival, dentistry is facing another challenge. With a mission to protect both patients and healthcare workers, we are adapting to the current epidemiologic situation and anticipate the incoming change. This article presents an unpublished prospective setting for dental care in the new chapter of pandemics after Omicron variant. Introduced biosafety protocol has been clinically tested for 18 months in the field. Three fundamental pillars of this sustainable biosafety protocol are: (1) UVC air disinfection; (2) air saturation with certified virucidal oils through nebulizing diffusers; (3) telehealth solutions. As a method of evaluation pseudonymous on-line smart form was used. This biosafety protocol is not relying on persons' identification as uninfected; it is designed for environments where healthcare workers or patients are hypothetical asymptomatic carriers. Results from 115 patient feedbacks suggest that with this protocol in place, there was no observed or reported translation of infection from patient to another patient or from patient to doctor or nurse and vice versa, albeit nine of the patients have retrospectively admitted being probably infectious during their dental appointment. The key of this protocol is its clinical sustainability, as full-body protective suits don't represent sustainable
\end{abstract}


dental care as well as there is no acceptable risk of patients getting infected during dental procedures.

Keywords: SARS-CoV-2; Omicron; Biosafety protocol; Dentistry; Orthodontics; Sustainability;

\section{Introduction}

Nearly two years have passed since the first stage of pandemics, where people refused to believe that there is a new virus that will spread and cause a pandemic around the world. Currently, there seems to be another denial phase of a pandemic facing the new Omicron mutations that are more transmissible than previous strains. Through the last two years, dental professionals were adaptable and smart to anticipate the risks of the incoming change. Since the appearance of the severe acute respiratory syndrome coronavirus 2 (SARS-CoV-2) causing disease (COVID-19) the biosafety measures at the dental offices have been revised repeatedly in effort to protect the patients and healthcare workers [1-10].

In general, the dental practice has been radically altered with COVID-19 pandemic. Dentists, especially during outbreaks, have changed their behavior and adapted their workflows [5,11,12]. Dental care is characterized by its close person-to-person contacts and treatment procedures that produce aerosols. Dental healthcare professionals - including dentists, dental assistants, dental hygienists and nurses were aware of high risk of exposure in the early stages of COVID-19 pandemic $[13,14]$. It is known that dentists have a high risk of contracting COVID-19 from their patients because of its transmission by respiratory droplets and the use of dental handpieces that can generate aerosols $[15,16]$ as well as the physical proximity to their patients $[14,17,18]$. Not all dental practices considered a transmission of SARS-CoV-2 as a real risk for them or their patient. Understanding the significance of aerosol transmission and its implications in dentistry can facilitate the identification and possible correction of negligence in daily dental practice [19]. Reviews focused on particles that can carry the virus and relevant approaches to mitigate the risk of pathogen transmission in dental offices often confirm high effectiveness of personal protective equipment in protecting patients and dentists from aerosols [20].

On the 16 of March, 2020, nearly 200,000 dentists in the United States closed their offices to patients in fear fueled by concerns that aerosols generated during dental procedures are potential vehicles for transmission of respiratory pathogens through saliva [21]. The findings, published 12 of May by Meethil et al. 2021 in the Journal of Dental Research [22] suggest lower risks for transmission of the SARS-CoV-2 virus during dental procedures than anticipated. These procedures are known to produce an abundance of aerosols. This fact has led to fears that saliva during a cleaning or a restorative procedure could make the dentist's chair a high-risk location. Meethill et al. have explored the origins of microbiota in aerosols generated during restorative procedures, ultrasonic scaling, and implant osteotomy by combining reverse transcriptase quantitative polymerase chain reaction (to identify and quantify SARS-CoV-2) and $16 \mathrm{~S}$ sequencing (to characterize the entire microbiome) with fine-scale enumeration and source tracking $[22,23]$.

As a conclusion, an asymptomatic carrier coughing in the dental office should be considered a much higher risk, than having an orthodontic attachment removed with a dental bur producing the aerosol. Clinical experience of authors of this article in the presented biosafety protocol is that preventive rinsing of patient can occasionally lead to coughing. This shall be preferably prevented in the space dedicated for dental procedures. While preprocedural rinsing has been encouraged since the beginning of the pandemic, guidance about which antiviral to use has been unclear. A number of rinses marketed to dental clinics have claimed that they are efficient in reducing or eliminating SARS-CoV-2 in the oral cavity. Indeed, the American Dental Association (ADA) initially recommended 
$1.5 \%$ hydrogen peroxide and $0.2 \%$ povidone iodine for use as an antiviral prerinse [24,25]. When actual antiviral testing of these commercial rinses on SARS-CoV-2 finally did become available, a different picture began to emerge. This new evidence challenged our widely held beliefs on the actual antiviral efficacy of several of these rinses.

At the same time when the ADA was recommending $1.5 \%$ hydrogen peroxide as an antiviral prerinse, the Centers for Disease Control and Prevention (CDC) was advising that $1.5 \%$ hydrogen peroxide needs 18 to 20 minutes to inactivate rhinovirus, the virus that causes the common cold [26]. An extensive literature review inspecting the antiviral efficacy of hydrogen peroxide mouthwash was published in the Journal of Hospital Infection in October 2020 [27]. After reviewing nearly 1000 publications that specifically assessed the virucidal effect of hydrogen peroxide, the authors concluded that: "there is no scientific evidence supporting the indication of hydrogen peroxide mouthwash for control of the viral load regarding SARS-CoV-2 or any other viruses in saliva."[27]. As a result of this research, and additional in-vitro and in-vivo testing, Public Health Ontario, the Royal College of Dental Surgeons and the Canadian Dental Hygienists Association have all advised their constituents to discontinue use of hydrogen peroxide as an antiviral prerinse [28]. The Antiviral Research Institute of Utah State University piloted a study in August 2020 of the antiviral efficacy of several oral rinses against SARS-CoV-2 [29]. Of all the tested rinses, $0.12 \%$ chlorhexidine gluconate and $1.5 \%$ hydrogen peroxide were inadequately effective, even after 60 seconds' exposure. While $0.2 \%$ povidone iodine did somewhat better, the only rinse to completely inactivate SARS-CoV-2 was a 100-ppm molecular iodine rinse. It was completely effective within 30 seconds. Neither of the iodine rinses were cytotoxic, but the hydrogen peroxide and chlorhexidine gluconate rinses were.

Currently, a molecular iodine rinse is the clear evidence-based winner as a prerinse for SARS-CoV-2. This is particularly important considering that a number of other oral rinses are neutralized in the presence of saliva [30-35].

Dental care is frequently provided to oncological or other immunocompromised patients that belong to a high-risk group. It was crucial that specific measures were implemented so these patients could be treated in a timely manner and in a safe environment. A timely adaptation of clinical workflows and implementation of practice modification measures was observed through the world [9,36-39]. These, with arrival of significantly more infectious omicron-strain, might need to be revised.

Dental care for cancer patients plays a crucial role in the symptomatic treatment and may strongly influence patients' quality of life. It includes prevention and local treatment in order to maintain adequate nutrition and provide reconstruction or rehabilitation after surgical procedures and radiotherapy [40]. Moreover, dental care is essential in dealing with toxicity of anti-cancer treatments such as oral mucositis, xerostomia, trismus, osteoradionecrosis, and opportunistic infections [41]. Active cancer has been recognized to have a possible negative impact on COVID-19 clinical course and outcome regardless of pre-existing comorbidities. Two-fold increase in death rate between cancer and non-cancer COVID-19 was observed, estimating that oncological patients represent a particularly vulnerable population [42]. Several studies discuss possibly lower protective effect and lower anti-SARS-CoV-2 antibody response rate after vaccination attributed to disease-related immune dysregulation and therapy-related immuno-suppression [43]. Optimal safety protocols should be applied to minimize the risks in this population during dental care.

After infection, SARS-CoV-2 can be detected in different body localizations. Dentists frequently deal with many of these sites in their daily practices. Since mass-vaccination the SARS-CoV-2 virus among asymptomatic individuals is less well-documented, but is important in future management of the dental environment $[38,44]$. The current data indicates that detection of SARS-CoV-2 and the infectious potential of the tested virus is dependent on the time of specimen collection relative to symptoms/infection and the presence of actual live viral particles [38]. 
Preventive bio-safety measures could take into consideration possible specific intraoral manifestation of COVID-19. Triad xerostomia, taste and smell dysfunction, and oral mucosal lesions are identified as common manifestations; however, the causality remains controversial. In the majority of publications regarding this topic there is consensual call for further observational studies focusing on this issue and on the causal relationships between oral lesions and COVID-19 [45-48]. Specific oral manifestations are observed in patients at Intensive Care Units (ICU), where prolonged prone positioning and mechanical ventilation devices were the primary risk factors for oral complications, in addition to the immunosuppressive drugs [49].

Growing evidence suggests that COVID-19 patients encounter several oral health problems such as dry mouth, lip necrosis, mucosal blistering, mouth rash, and loss of taste and smell. Periodontal disease and severe inflammatory gum disease may worsen the symptoms associated with COVID-19. Routine dental therapy may help decrease the symptoms of COVID-19 [50].

Pandemics had a significant impact not only on dentists and their colleagues [51], but also on patients' mental well-being. Frequently, the occurrence of depression, anxiety, stress, intrusion, avoidance, and hyperarousal were observed both in patients, as well as in healthcare workers [52-56]. In the beginning of the pandemics the Hospital of Stomatology in Wuhan diagnosed nine dental staff members infected from $23^{\text {rd }}$ of January to $4^{\text {th }}$ of February 2020 [14]. Chinese dental surgeons responded with recommendations for the management of dental care workflow in the context of the epidemic [17]. Since then, various recommendations and guidelines have been published on professional websites in many countries. For example, in the US (Centers for Disease Control and Prevention (CDC), American Dental Association), in Europe (European Centre for Disease Prevention and Control (ECDC)), in France (Health Ministry, French Dental Association), in the UK (National Health Service, British Dental Association) [57]. One of the first renowned pandemic-dental events in European dentistry was the outbreak in North Italy in Lombardy. All of the Lombardy's dentists were evaluated with an online ad hoc questionnaire. 3599 questionnaires were analysed. $502(14.43 \%)$ participants had suffered one or more symptoms referable to COVID-19. Thirty-one subjects were positive to the virus SARS-CoV-2 and 16 subjects developed the disease. Only a small number of dentists $(n=72,2.00 \%)$ were confident of avoiding infection [58].

Many new approaches of dental diagnostics or treatment management were introduced and various innovative trends were strengthened - for example tele-health solutions like Dental Monitoring ${ }^{\circledR}$ (DM) (Dental Monitoring Co., Paris, France) [59-65].

Moreover, the dental professionals in the early efforts to adapt their biosafety measures performed typically a web-search conducted in the main databases of the scientific publications. This early research often led to possible revisions of biosafety and disinfection protocols at the dental offices [5,12].

The ongoing pandemic has changed the practice of dentistry forever, some of the changes have made dental care more time-consuming, difficult and costly due to the possible pathways of transmission and mitigation steps needed to prevent the spread of the infection. Despite the widespread anxiety and fear of the devastating health effects of COVID-19, only $61 \%$ of dentists have implemented a fundamental modification to their treatment protocols. Facing the highly infectious Omicron strain, as an urgent matter of public health, all dentists must identify the additional steps they can take to prevent the spread of infection [16]. A clinical practice guidelines used in the first year of pandemic might change with a new more infectious variants. The clinical practice guidelines developed during the first year of the pandemic offer recommendations which guide dental staff in providing safe dental care, minimizing exposure to SARS-CoV-2 and reducing the risk of COVID-19 infection in the clinical environment. Such recommendations must, however, be updated as new evidence arises [47]. There is a high level of agreement between different dental specializations about the necessary preventive measures of the routes of transmission. Published data regarding the survivability of the virus on innate 
objects vary substantially [67]. Nevertheless, due to the wearing of personal protective equipment (respirators, gloves, masks, eye shields, and gowns) and use of disinfection procedures, this risk can be significantly mitigated. Research published by Estrich et al., 2020 found in June 2020 through web-based survey that during the first wave of pandemic only $0.3 \%$ of surveyed dentists had a probable COVID-19 diagnosis, from these $82.2 \%$ were asymptomatic. The most commonly reported health problems among dentists during pandemics were anxiety and depression $[16,68]$.

The primary aim of most biosafety protocols is to prevent any cross-contamination while allowing the provision of urgent and emergency dental care. Aerosol-producing and other elective procedures should be avoided in the periods of outbreaks of unknown variants [11,69-72]. Various biosafety protocols are detailing the safety and operational measures to be taken, while providing the dental care in the COVID-era. Falahchai et al. 2020 published a comprehensive protocol regarding dental care during the COVID-19 outbreak. The point in the outbreaks caused by new, not well researched variants, is that these might bring dangerous long-term health hazards enabled by new mutations. During these short periods, the dental treatment might be limited to patients with urgent or emergency situations. Separate waiting and operating rooms should be assigned to patients in order to minimize the risk of infection transmission and the treatment should be provided with the same protective measures with regard to Personal Protective Equipment (PPE) for the dental clinicians and staff [73].

Pandemic situation in dental care and the attitude towards it have been varying during the last two years. From initial negligence of possible serious impacts of the virus spread, up to panic precautions. After a year, the variables of the pandemic situation have changed with the implementation of vaccination. This represented an important milestone $[74,75]$ suggesting a possible end-game scenario for the COVID-19 pandemic. These expectations are facing disappointment with the arrival of the Omicron variant. Imaginable race between SARS-CoV-2 mutations and vaccine rollouts was slowed down with negative perception of people afraid of possible vaccination side effects. Recent study from Czech Republic proved the distribution of side effects among Czech healthcare workers as highly consistent with the manufacturer's data. The overall prevalence of local and systemic side effects was higher than the manufacturer's report [75]. Data suggest that Omicron capabilities to evade natural human immunity, protective effects of distributed vaccines $[76,77]$ and its resistance to current monoclonal medication $[78,79]$ make the Omicron variant a true dilemma. According to a recent study by Dr Michael Chan Chi-wai, from 15th of December 2021, it can infect faster and better than Delta in human bronchus, but with less severe infection in lung [80]. Near future will provide the answers considering the strongest attributes of this variant as they possibly are milder clinical manifestation or higher infectiousness. However, its newly gained strong ability to infect ACE2 in mice predicts this variant is here to stay until pushed out with more infectious strain [81].

Recent study of American dental association and research published by Araujo et al., 2021suggests, that US dentists continue to show a high level of adherence to enhanced infection control measures in response to the ongoing pandemic, resulting in low rates of cumulative prevalence of COVID-19 [18]. COVID-19 infections among practicing dentists will likely remain low if dentists continue to adhere to guidance. With the spread of "Omicron-like" mutant strains the likelihood of recurrent infection raises some doubts on whether vaccination alone will provide long-term immunity against COVID-19 and its future variants. Furthermore, several mutations in the receptor binding domain and S2 are predicted to impact transmissibility and affinity for ACE-2 that might be relevant for adaptation of biosafety protocols [82]. From this perspective, the aim to provide biosafe dental workflow in orthodontic or any other dental practise shall take into account higher transmissibility of the virus and higher number of asymptomatic carriers. Anticipation when the virus spread naturally diminishes or becomes less virulent is not possible neither is certainty that further mutations will be harmless. This is why the effective biosafety protocol shall be sustainable. Based on these facts, various simulations and modelling 
data, dental practices may never return to "normal", former routine operation even after global vaccination would be somehow successful as there would still be a significant risk of outbreaks of infection with new mutant strains. Variable, multi-level measures will still be required, depending on the local COVID-19 cases rate, to secure safe dental care provision [37].

In the past, dental care was routinely provided without any protective gear, however after 1991, dental personnel were required to wear gloves, masks, gown, and protective eyewear. Dentistry is one of the most exposed professions to respiratory diseases e.g., COVID-19. Initial patient screening can be nowadays performed even before the patient meets the dentist in person. The dentist's review of the patient's medical history is mandatory at the beginning of every clinical appointment [83].

This paper is introducing a field-tested clinical biosafety protocol focused on orthodontic workflow in confrontation with the incoming Omicron variant that is very invasive against natural immunity whether it's vaccine or previous infection [76,80-82,84]. However, this variant isn't more invasive in any degree against masks, and indoor biosafety measures.

Biosafety protocols applied a year after pandemics outbreak were not significantly different from the ones that are effective today. The Delta variant, despite being more infectious, did not require any fundamental remake of the biosafety protocols in dental offices.

Omicron SARS-CoV-2 (B.1.1.529) variant with its already researched properties and some anticipated ones can be considered a new chapter in the COVID-19 pandemic. This highly-mutated strain has more than 30 mutations, several of which overlap with those in the Alpha, Beta, Gamma, or Delta variants of concern. These mutations and deletions are known to lead to increased transmissibility, higher viral binding affinity, and higher antibody escape. The infectious potential of the new variant is not certain yet [76-78,81,84-88] and preliminary research publications suggest it is from 2 to 4,2 times more infectious than Delta variant $[84,89]$. It evades not only human natural immunity as well as it evades immunity from vaccines [81] and as number of laboratory studies have previously shown a reduced ability of approved vaccines to neutralise the new variant, the picture on treatments had been less clear. A bibliographic review of the main sources of information including MEDLINE (by means of PubMed), Scopus, Science Direct, SCIELO, and Google Scholar confirmed frequent use of rinses, aerosol control, hand washing and some other precautions in regard to dental care $[6,12,90]$. The most dangerous period of infection in dental office is the early asymptomatic stage. The omicron variant transmits more, and escapes immunity built naturally. Hundreds of researchers globally are racing to understand the new variant, which is the most differentiated strain yet among the five variants of concern identified by the WHO since the pandemic began [89].

People infected with Omicron variant might have clinical symptoms and significant viral load despite hight titres of neutralisation antibodies. This could mean that original antibodies do not ensure sufficient defence against Omicron infection. $4^{\text {th }}$ and $5^{\text {th }}$ shots of vaccine based on the same antigen (spike protein) might not be path to safe future as a terminal solution of covid pandemics. It is important to realize that development of either multicomponent vaccines or vaccines against currently circulating virus variants can bring an effective last line of our defence. Vaccines work after the virus has entered the body. The safety protocol presented in this article provides multilevel system of defences as mostly airborne protections.

On 26th November 2021 the Technical Advisory Group on SARS-CoV-2 Virus Evolution was called to assess the new SARS-CoV-2 omicron variant labelled as B.1.1.529, was firstly announced from South Africa on 24 November 2021 [62]. Soon there were identified more than 100 cases across the whole world until November 26th - in Hong Kong, Israel, Italy, Belgium and Egypt [63]. It was presumed that the variant was already circulating in communities since the case in Egypt had not epidemiological connection to cases in South Africa [64]. The latest coronavirus epidemic peak in South Africa was caused mostly by 
Delta variant, rising surprisingly higher closely before omicron variant announcement. South Africa Health Department presented that B.1.1.529 variant is becoming dominant in their population [65], suggesting high transmissibility. This new announced variant was assigned by World Health Organization (WHO) as Variant of concern (VOC) which means that it might have increased transmissibility, virulence, clinical disease presentation or changed epidemiology, decrease in diagnostic, preventive or therapeutic possibilities. WHO recommended countries for enhanced sequencing and submitting the genome results to publicly available databases, monitoring and reporting detected cases[62]. WHO has been a respectable authority and one of the most trusted information sources among students in the face of fake news and misinformation accompanying the pandemics [94].

The ability of this variant to evade immunity from previous infections in contrast to Delta and Alpha variants was presented in population-level evidence [88] and thus make this suggestion epidemiologically important in countries with huge amounts of previously infected cases, as Slovakia is. An increased risk of reinfection of omicron variant is known according to preliminary evidence [62],[66],[67] and probably caused by Omicron escape of neutralization antibodies [97]. Even after two doses of mRNA vaccines, higher transmissibility of Omicron was observed also in Norway [98]. The booster dose may not prevent getting infected, but observed symptoms were mild or moderate [99-101]. The time since second or booster vaccination plays role in effectiveness against symptomatic disease, as noticed in United Kingdom [102].

Preliminary results from South Africa still show $70 \%$ efficacy of two doses of BNT162b2 vaccine against hospitalization, the explanation could be based on population immunity with induced T-cell immunity, gained after infection or vaccination $[103,104]$. The data from Israel and United Kingdom highlights the need of booster dose to reduce hospital admissions [105].

Several countries introduced travelling restrictions to South Africa, nevertheless other countries are reporting new suspected cases and the dominancy of Omicron variant in the countries with time, for example the Czech Republic which is a country closely related to Slovakia with tight citizen relationships. The Slovak Ministry of Interior announced the duty of reporting and home-isolation after travel from South Africa, Israel, Hongkong or Seychelles and promised priority sequencing of PCR swabs from cases with such travelling history.

Based on these preliminary evaluations of Omicron, each new VOC results in uncertainty - struggling about transmission reduction, discussing vaccines efficacy, supplying elective-care and preventing long COVID complications. Thus, not only vaccines play a key role in preventing COVID-19 spread, solutions such as biosafety protocols are complementary and highly demanded.

The risk for transmission of pathogens in a dental office resulting in an infectious disease is still unknown; it seems to be limited in developed countries but it cannot be considered negligible [106]. The literature review revealed five distinct areas of pandemic control, comprising (1) planning and protocols, (2) patient screening, (3) preparation of facilities, (4) PPE and infection control, and (5) practices and aerosol control; thereby covering systematically all aspects needing adaptation in a pandemic context [107].

Research published by Estrich et al. 2020 showed that dental professionals have enhanced their infection control practices in response to COVID-19 and have benefited from greater availability of personal protective equipment. In this research, after the first pandemic wave, most of the dentists (82.2\%) were asymptomatic for 1 month before administration of the survey. Enhanced infection control procedures were implemented in 99.7\% of dentists' primary practices, most commonly disinfection, COVID-19 screening, social distancing, and wearing face masks. Most practicing dentists (72.8\%) used personal protective equipment according to interim guidance from the Centers for Disease Control and Prevention [68]. As the pandemic situation will develop towards more infectious variants, new considerations, new protocols, and new mechanisms will be implemented in the dentistry profession because of the past experience of the COVID-19 pandemic. There is high 
probability that many more procedures will take advantage from tele-dentistry approach [108].

Possibly lower lethality of Omicron variant in combination with significantly higher ability to infect and create asymptomatic carriers might be dangerous. COVID-19 is not only about deaths from its pulmonary form. The effects of COVID-19 are very diverse and vary from individual to individual. SARS-CoV-2 infection can lead to severe acute respiratory disease and multisystem failure. Many patients, especially after treatment at the ICU, develop long-term disabilities, such as pulmonary, cardiovascular, hematological, renal, gastrointestinal, reproductive, psychological and the central nervous system problems, that can last from months to years [109]. SARS-CoV-2 enters the body through the upper and lower respiratory tract, indicating the most common manifestation - COVID19 pneumonia. Acute lung damage can lead to additional long-term pulmonary malfunction [110-112]. The virus can travel also to the other organs and the ACE2 receptor on the cells serves as the enter point for the virus. Moreover, hyperactivation of the immune response can cause other health issues. The olfactory pathway or the blood-brain barrier pathway can let the virus into the neural system and cause neuronal damage as well as neurodegeneration or long-term neurological and psychosocial consequences [112-115]. Cardiovascular problems (e.g., myocarditis, arrhythmias, myocardial damage) are quite common too [116] and recent studies have shown that SARS-CoV-2 infection also affects the human reproductive system, especially the male reproductive system also via the ACE2 receptor $[117,118]$. Despite our natural wishful thinking, hopes for Omicron to be turning into harmless flue might be disappointed, as this process might take generations or may never happen. Even if Omicron does cause less severe disease, the sheer number of cases could once again overwhelm unprepared health systems. It carries a higher risk of re-infection and asymptomatic carriers, so it is just matter of time until it will reach the dental offices. It is up to us if the dental care will remain safe and sustainable at the same time.

This article introduces an unpublished novel set of biosafety measures and dental workflow adaptations to preserve the biosafety in dental care. This biosafety protocol could possibly provide a simple and sustainable model for a biosafe dental workflow based on combination of various technologies. It shall handle renewed and increased risks brought by more infectious Omicron variant carried with asymptomatic infectious patients.

\section{Materials and Methods}

\subsection{Main objective}

The main goal of this paper is to introduce a comprehensive biosafety protocol for dental care as a prospective setting for new era of pandemics with Omicron variant.

\subsection{Participants and the environment}

The protocol was applied and evaluated in clinical environment of dental clinic with 4 doctors, 4 dental nurses and one manager.

The clinic has two working shifts per day and is open six days per week including Saturdays.

During period of protocol evaluation two working shifts took place from 6.00-14.30 and 13.00-21.00.

The first and last 90 minutes of the day were dedicated to thorough disinfection procedures of surfaces, tools, chairs in the clinic. Each Friday a total deep sterilization procedure of dental units was scheduled.

During eighteen-month evaluation period approximately 2500 appointments were scheduled in digital calendar for 160 different patients not including their accompanying 
persons. Approximately 2230 appointments took place (other were cancelled or postponed mostly due to pandemic health precautions or travel complications).

The environment for dental care consists of the fully separable rooms with independent air processing and separate Ultra Violet - C light (UVC) air sterilization systems. Each of three rooms has an independent dental unit with separate nurse position.

\subsection{Brief description of the protocol}

The key attributes of this biosafety protocol are:

- Efficient

- Sustainable

- Simple

- Applicable in other dental specialties (despite orthodontic customization)

The fundamental backbone of this protocol is a combination of air treatment and new technologies. Air treatment is performed with combination of hooded UVC sterilisation and air disinfection with creation of virucidal air puffer made by certified virucidal oil nebulizer/diffuser. Key technologies implemented as part of this protocol in the workflow are: artificial intelligence in patient diagnostics, continuous and post-treatment monitoring, smart mobile patient coaching, 3D printing for aerosol control and some other techs.

By someone, the set-up of this protocol might be considered as a biosafety overkill, however the risks of airborne infection that could come from an asymptomatic carrier and circumvent immune defences causing an irreparable health damage to someone else, is not negligible.

This working protocol is intended as a possible complement to existing methodologies. It represents more than a year of our efforts dedicated to clinical testing and the implementation of various new technologies and working procedures in order to maintain the biosafety of dental healthcare. Dental care must be provided with regard to the patient safety as well as safety of healthcare professionals.

This protocol was created in interdisciplinary cooperation of epidemiologists, infectologists, dental surgeons, general dentists, orthodontists and other healthcare professionals.

We believe that the protocol provides inspirational potential for wider clinical implementation. It is our contribution to solving this historical situation affecting safety and availability of oral care.

The fundamental principle of this protocol is full room volume air treatment with UVC and virucidal oil diffusion. Sterilization of the entire volume of air (for each patient) of the given treatment unit (room) by a combination of UVC light with a created permanent virucidal buffer of certified disinfectant oils and special active FFP3 protective aids. The key additions to the protocol are fundamental changes in some working practices in the provision of artificial intelligence and telemedicine.

There are two main parts of the permanent daily protocol:

- $\quad$ Full room-volume air UVC sterilisation for each patient *total air volume per dental unit (separated room)

- Permanent air dispersed super small droplets of virucide oil - a protective puffer in recommended concentrations, created with disinfectant fogging machine (nebulizer)

There are other complementary technologies we have introduced for our workflow:

- AI video-scan evaluation (common checkups rendered obsolete) - Dental Monitoring

- custom made IOS/Android app coaching the proper habits - StrojCHECK [4]

- 3D printed aerosol vacuum pump ending, that supports aerosol dispersion during dental procedures and other customized $3 \mathrm{D}$ printing allowing more 


\subsection{Comprehensive Biosafety Protocol Description}

\subsubsection{Introduction of the protocol}

Orthodontics is a specialized department of dentistry that has its own specifics and in some forms of provided healthcare differs from the procedures of other dental care. It focuses on the prophylaxis and therapy of anomalous teeth, jaws and intermaxillary relationships and is most often perceived by the average patient as "tooth straightening treatment". Like other areas of dental care, and in fact all areas of medicine, we have encountered the need to find a comprehensive and sustainable way of caring for patients.

The aim of this protocol was to define the safest and at the same time physically and economically sustainable measures for a longer period of the pandemic with the minimization of the impact on the provided health care in dental practice. The aim was not to reduce the quality of care provided, not to increase its cost and not to tolerate the risks of exposure of staff or patients.

Due to the number of unknowns around the current infection and especially its longterm consequences, we do not consider it responsible to allow even a low-risk of exposure to our patients or healthcare professionals.

We began work on this protocol on the $6^{\text {th }}$ of February 2020, and it took us more than 9 months to experiment, test, and implement various new practices, technologies, and modifications to existing practices. Through final consultations with experts, it has taken on its present form. We currently consider this protocol to be useful and inspiring for clinicians looking for cost-effective solutions that can be sustainable in the long run. The aim of this protocol was to adapt and introduce new forms of healthcare provided so far for the clinic providing specifically orthodontic dental care and dental hygiene. The premise is to safely treat a seemingly healthy but infectious patient with minimal risk of infection for the healthcare staff and other patients being treated. Equally important is the protection of the patient from an asymptomatic but infectious health care provider. Despite all our staff is fully vaccinated, with Omicron variant, they might be infectious and without clinical symptoms at the same time [76-80,84-88].

In order to develop this protocol, we have implemented new and improved several existing procedures in cooperation with renowned infectologists, epidemiologists and other experts. Example of some implemented technological innovations:

1. Smart-tracking mobile application (eg https://www.zostanzdravy.sk/ (accessed on 1 December 2021) and its successors [119])

2. On-line dynamic anamnestic forms (effectively replacing part of 4D clinical examinations)

3. Tele-dentistry Tele-orthodontics - Dental Monitoring® (DM) (Dental Monitoring Co., Paris, France) / https://dental-monitoring.com/ (accessed on 1 December 2021) [120] and (StrojCHECK®, Bratislava, Slovakia, 3Dent Medical, www.osim.sk (accessed on 1 December 2021) [4]

4. Artificial intelligence in tele-diagnostics - in active screening of patients in tandem with the doctor

5. Special medical devices - active FFP3 shield respirator - BioVYZR - Toronto USAbased Vyzr Technologies, a shield that covers the wearer's face and protects against droplets and pathogens. Powered Air Purifying Respirator (PAPR), a device typically used only in industrial and healthcare settings.

6. 3D printing of sterilizable devices - (aerosol aspirators for surgical aspirators or individualized handles for drapes from 3D face scan - Lidar scanner in iPhones)

7. Two-phase air sterilization (diffusers of biocidal oils with UVC, NewAroma.sk) 


\subsubsection{The protocol}

This protocol does not inevitably change the procedures already established so far for sterilization of dental instruments, disinfection of surfaces and other routinely applied rules of each dental clinic. These remain in force and their possible tightening is described below. Covid is just one of the many infections we fight in asepsis in the dental clinic. Its key set of differences from HIV, herpes or other common viruses is the relatively long period of possible asymptomatic infectivity. The advantage of modern orthodontic therapy with clear aligners is the relatively rare occurrence of the need for urgent treatment. In the case of urgent orthodontic treatment of a confirmed infectious COVID-19 patient, a bio-hazard protocol is applied as in HIV patients, in COVID19 with an emphasis on the prevention of airborne spread. Regardless of whether the treatment involves aerosol dispersion (e.g., removal of attachments or fixed retainer or orthodontic auxiliaries like distalizer, power-arms [121,122] or power caps), the treatment procedure differs from the standard protocol only in the fact the patient has the last appointment of the day shift - as the last procedure on a given day.

Oral rinsing is not routinely recommended in our protocol for all patients. Only in the patients where intra-oral procedure is planned, a iodine prerinse for 30 seconds is indicated. Rinsing is not addressing the nasopharyngeal region with the largest "virus load" (reported in the nasopharynx). From our practical experience, the recommended 30-second rinsing often led to a "run-in" and subsequent coughing of the patient and thus unnecessary contamination of the space. This protocol only recommends rinsing with careful rinsing only during attachments replacement or cleaning, bio3dir removal and "powerCaps", "powerArms" removal, interproximal recontouring or dental hygiene procedures. Prior to these procedures, the patient rinses the mouth for 30 seconds.

This protocol works with the premise that either patient, nurse or doctor are infectious. This is the reason why as a part of the protocol is introduced:

1. minimization of time exposure (shortened duration of treatment, replacement of unnecessary physical visits of the patient via AV technologies, ordering for the exact time with preparation of everything necessary for the procedure in advance)

2. mechanical and physic-chemical prevention in the dispersion of the virus and its carriers into the space resp. decontamination (PPE, continuous sterilization of the air and virucide "puffer" in the air), et ...

3. preventive (patient input filter - symptoms, smart-App tracking, continuous testing of healthcare professionals *)

Most orthodontic procedures are short-term and therefore asymptomatic patients are treated. For example, a slightly elevated temperature that is acceptable under this protocol is $37.8^{\circ} \mathrm{C}$ (originally taken from CDC). The uniforms of the medical staff are rotated on a daily basis. They include all surface clothing including shoes and socks.

\subsubsection{Technologies in the protocol}

The protocol implements the following technologies and practises:

1. pandemic sentinel smart-apps

2. Online dynamic anamnestic forms

3. Artificial intelligence in dental monitoring

4. Robotization of automatic in surface disinfection

5. A.I. smart patient app for treatment coaching

6. 3D printing of sterilizable devices

7. Regular COVID testing of personnel from saliva tests

8. Testing of loss smell and higher temperature

9. UVC and diffused virucidal oil air treatment:

10. FFP3 shield active respirator 
1. Patient input filter by means of intelligent pandemic sentinel apps. Authors participated on development of such an intelligent pseudonymous app that would alert its user when network would register a clinically relevant exposure to another pseudonymous installation defined as infected. Use of smart tracking mobile applications e.g., mobile application www.zostanzdravy.sk (Figure 1), was available at the beginning of March 2020 on the Android platform. (IOS blocked it). It exceeded 200,000 installations in the Slovak Republic, albeit never received official support from government. Later in cooperation with Sygic company it continued as "Stay Healthy - COVID19". It was handed over to the government of Slovak Republic free of charge. Unfortunately, such an app was never officially launched, A patient actively using such a smart application would represent a lesser threat to the clinical environment, and this type of application is one of the layers of the patient input filter suitable for urgent treatment. These types of smart applications are frequently used in many countries and are useful layer in biosafety management.

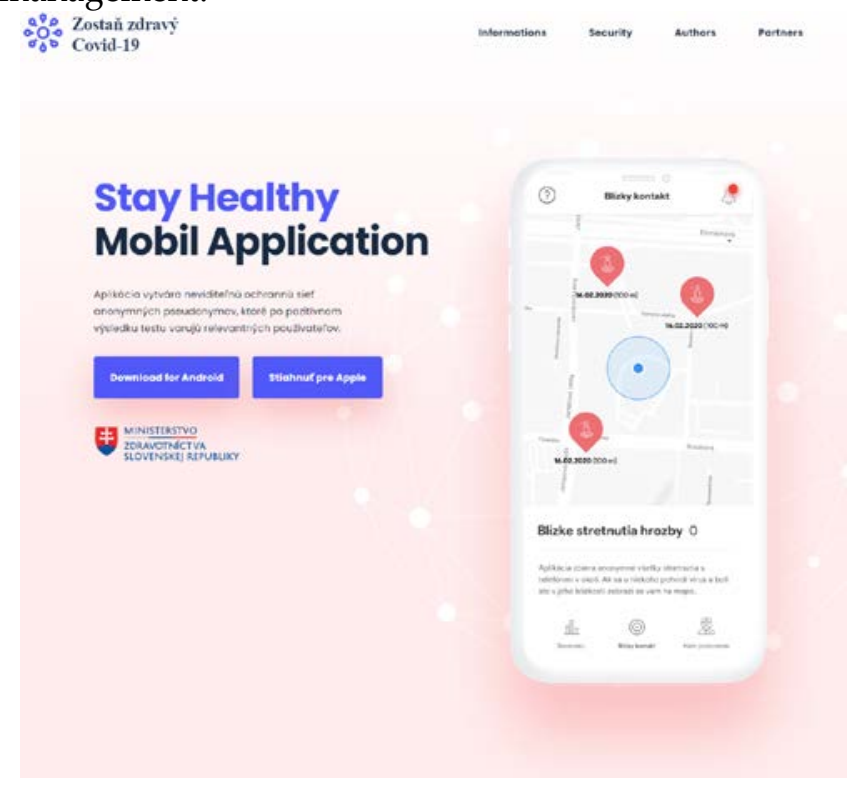

Figure 1. Screenshot of smart application for SARS-CoV-2 infection tracking, retrospectively alerting a pseudonymous installation when exposed to another "infected" pseudonymous installation of this app. As indication for getting a PCR test. Healthy Mobil Application - never acquired full governmental support and until today there is not such an official app rolled out. www.zostanzdravy.sk/en [119]

2. The use of smart forms like Typeform - www.typeform.com brings interactivity to communication with patients at home. Online dynamic anamnestic forms: use the patient's own mobile phone not only for video communication (WhatsApp, facetime), but also for outsourcing part of the examination, for example with the right instructions, a short video sequence of a natural smile captured by the patient is more natural than formulate on the basis of ongoing responses eliminates the need for physical interaction. The anamnesis itself is very important in reducing the risk of transmission, the proportion of asymptomatic patients with COVID-19 is less than $30 \%$ in the number of children with the highest proportion of asymptomatic infection. Identification of symptomatic patients is the first and relatively effective method of risk reduction. Due to the fact that the anamnesis is taken remotely, neither staff nor co-patients are exposed to the risk of infection, nor is there any contamination of the surfaces in the waiting room $(2,3)$. We use dynamic forms from Typeform company for this protocol. Remote screening of COVID-19 symptoms by application has been identified as a suitable method for 
detecting COVID-19 infection. Even the survey form for this biosafety protocol clinical evaluation was created as a smart form. Asking a set of questions that differs according to the answers. Logic behind is shown on Figure 2.

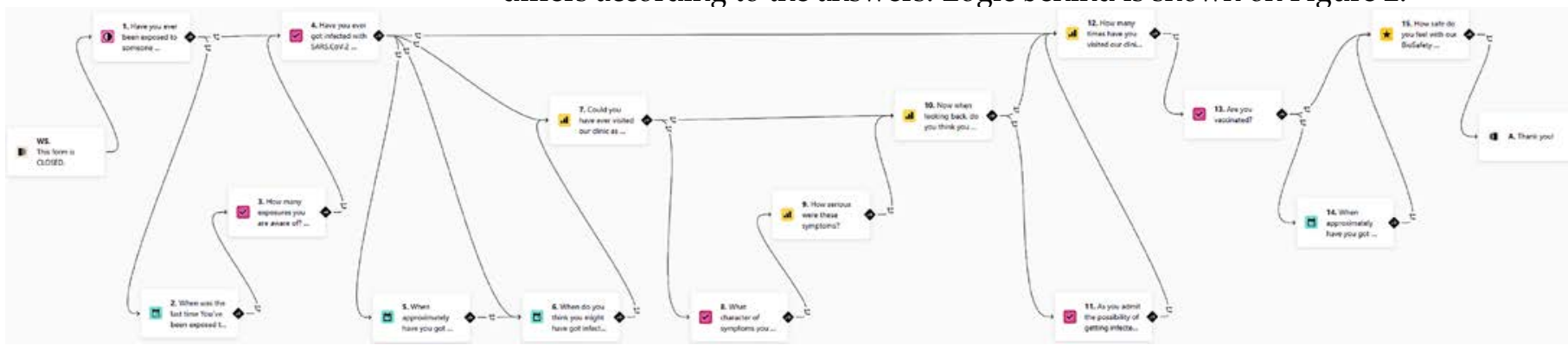

Figure 2. Example of logic behind the Smart on-line form - English version of survey evaluation this protocol. URL: https://sangreazul.typeform.com/BioSafety-ENG (Accessed on 15th of December 2021) [123]

3. The artificial intelligence used in the Dental monitoring software is using the patient's mobile phone for regular "scanning" either to assess the course of treatment or screening a growing patient with monitoring of development or. monitoring retention stability after cessation of treatment. A special holder allows you to record a video of your own teeth. It allows paradoxically more regular and even more thorough inspection using A.I. Artificial intelligence allowed us to summon the patient to an appointment only when necessary (Figure 3). Each video scan first evaluates A.I. and alert the doctor only to the monitored situations. The form of our workflow has thus changed fundamentally, and healthcare professionals spend a large part of the day reviewing the outputs of artificial intelligence, which in turn extremely efficiently evaluates huge volumes of data video scans of our patients' oral cavities. It is not humanly possible to evaluate the hundreds of video scans that patients regularly make. The "brute force" of this technology is ideal for identifying situations requiring intervention. Similar to this artificial intelligence application another application can differentiate cough of the patient identifying the cough specific for COVID 19.

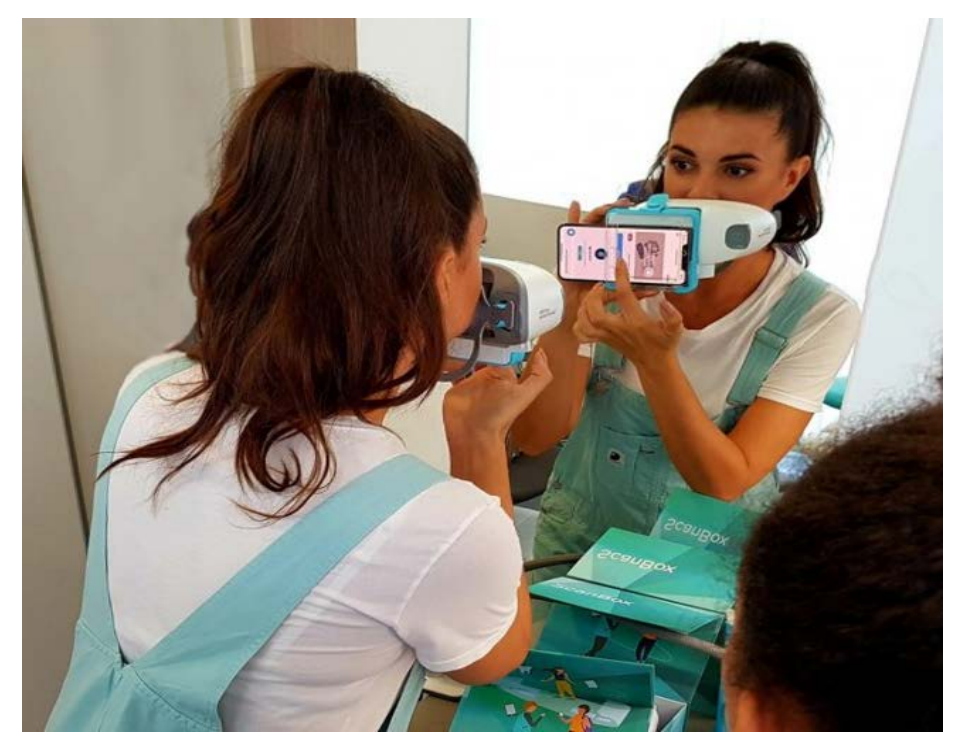

Figure 3. First Dental monitoring session is patient educated with the nurse how to use the free app in his/her own mobile in combination to "scanbox" holder. Published with written consent of the person. 
4. Surface disinfection in clinical practice during pandemics is perpetual task. It should respect all existing guidelines with an emphasis on consistency and differentiation of surfaces and a higher frequency of cleaning (after each patient). After entering, the patient shall be in contact only with the necessary surfaces, first disinfecting her/his hands. Frequent cleaning of surfaces in the clinic, after each procedure, should include frequently inspected surfaces such as handles, keyboards and mice. Addition of floor cleaning by robotic vacuuming with disinfection mop - in our case iRobot Braava jet $\mathrm{m} 6$ suitable for up to $100 \mathrm{~m}^{2}$ (Figure 4). The CDC recommends applying standard virucidal disinfectants to potentially contaminated surfaces to prevent the spread of SARS-CoV-2 infection. The work on the susceptibility of human coronaviruses and SARS-CoV is expected to be highly effective in SASRS-CoV-2that is relatively resistant to environmental conditions and remains infectious on smooth surfaces such as metal and plastic for many days.

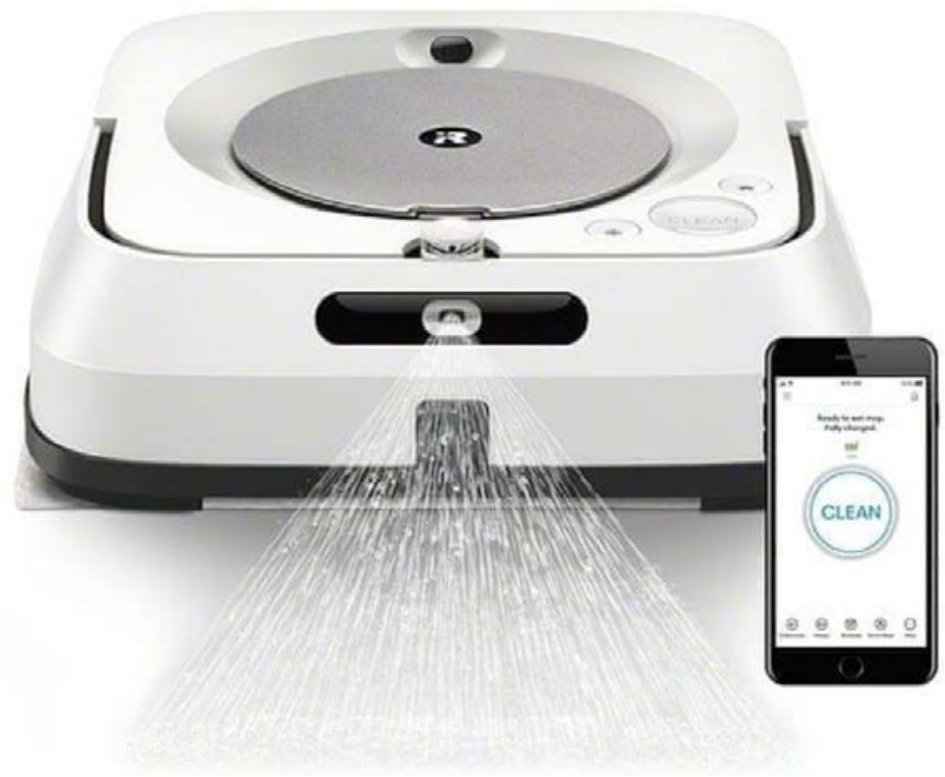

Figure 4. Robotized cleaning of surfaces like floor after each procedure can free the professional staff for other duties. Using smart robots managed with mobile application proved useful during pandemics.

5. Self-developed smart mobile applications for patient treatment coaching and remote discipline support (Figure 5s). Authors of this paper have been gradually developing iterations of the smart patient application for patients in orthodontic treatment with clear aligners, to support the proper habits - stereotypes. Such an app allows better remote management of the patient and possible reduction of the frequency for visits in the dental office. The application educates and motivates the patient to behave responsibly [4]. For example, the app provides motivation and coaching for more frequent cleaning of teeth and aligners and in general it approved the patient compliance. 


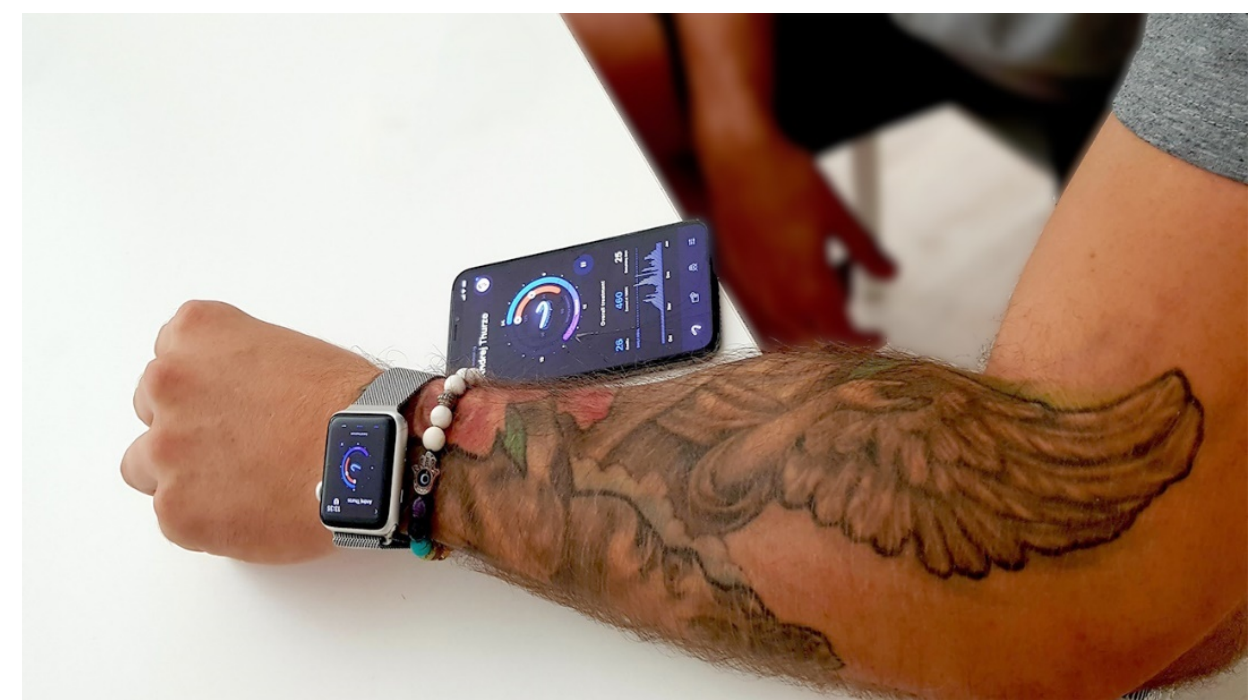

Figure 5. Patient smart mobile app developed by doctors for their patients improves compliance of patient during pandemics and perform automatized coaching.

6. 3D printing of specific sterilizable devices for example, an aerosol aspirator (Figure 6). This device is printed by a Multijet Fusion 3D Pro Printer. It has been developed by an Italian orthodontist because of Coronavirus. This aerosol interception device is called "SUR-FACE" and is sterilizable at $121^{\circ} \mathrm{C}$ in autoclave. It connects to conventional $16 \mathrm{~mm}$ suction device. The material is polyamide and is compatible with any retractor with thickness $\leq 2 \mathrm{~mm}$. The material is recyclable and supplied with 5 additional rubber seals. Due to its shape, this aerosol aspirator is clinically valued as a very effective tool for aerosol management and naturally is considered as one of the potentially most contaminated work aids. The long service life of SARS-CoV-2 on plastic surfaces has been demonstrated. The one-time use of suction cup attachments minimizes the risk of transmitting infection with this tool. The sterilizability of these handpieces is crucial. 

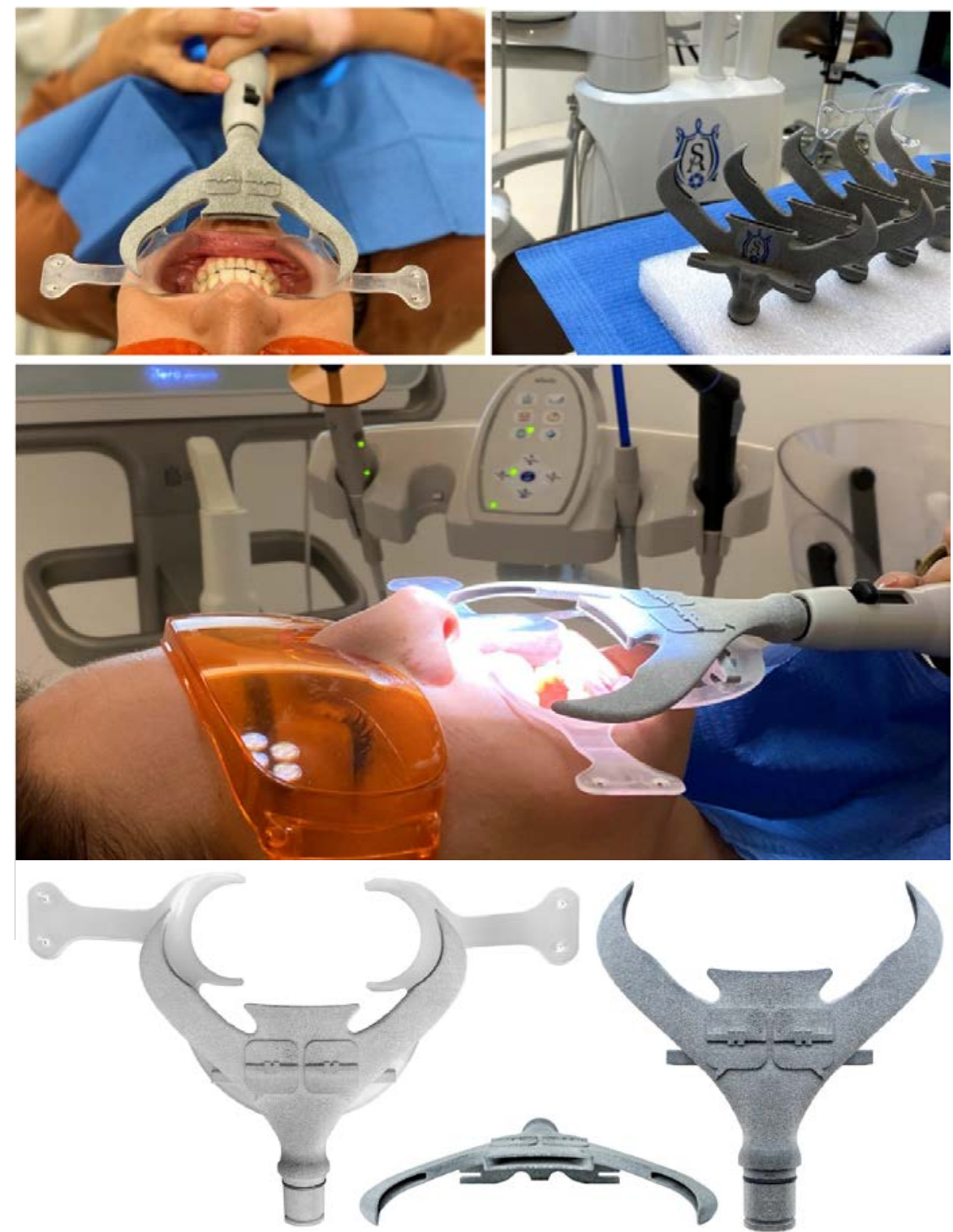

Figure 6. This aerosol interception device is called "SUR-FACE" developed by an Italian orthodontist because of Covid-19 pandemics. It is printed by a MultiJet Fusion 3D Pro Printer and is sterilizable at $121^{\circ} \mathrm{C}$ in autoclave.

7. Regular COVID testing from saliva tests or other convenient form of testing for possible infectiousness of everybody in the team, shall be employed as with arrival of Omicron variant it is even more likely that a healthcare professional will become a "supercarrier."

8. Testing of loss smell and higher temperature $\left(37.8^{\circ} \mathrm{C}\right)$ and signing the patient's affidavit are part of a routine patient filter. Patient aware of his/her exposure is reminded about the responsibility, as well this primitive entry filter is a space for informal communication between patient and nurses further researching possible exposure. We have made samples from food essences; coffee beans are just a decoration and possible association for someone without smell (Figure 7). Anosmia - Loss of smell manifests itself to varying degrees in most COVID patients and is usually temporary. It is not yet confirmed with Omicron infections. Anosmia is ten times more likely with COVID than fever, cough or respiratory problems. 


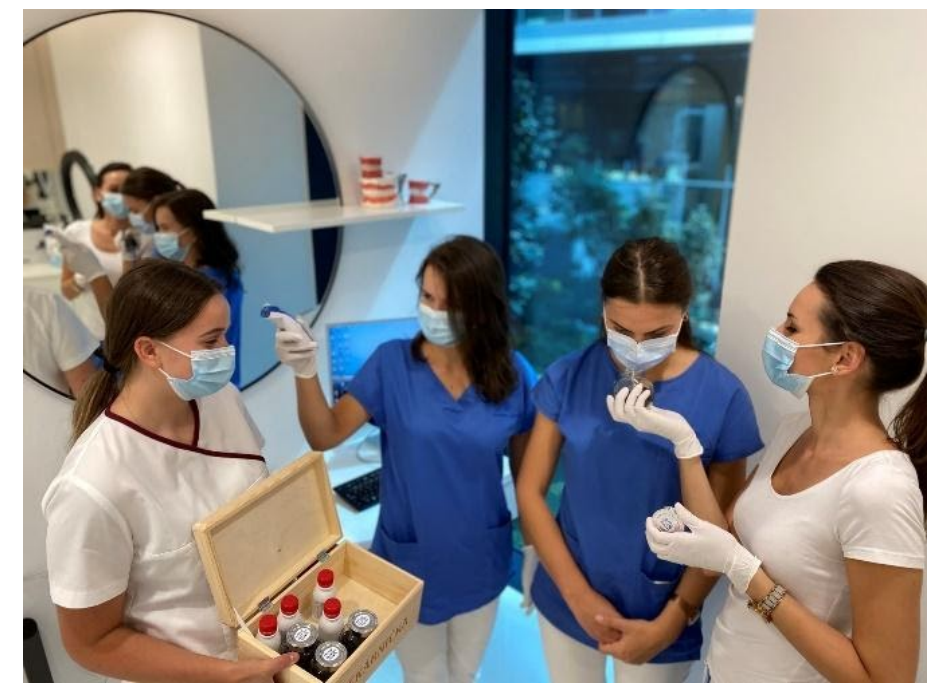

Figure 7. Testing of loss smell and fever with signing the patient's affidavit are part of a routine patient entry filter.

9. Two-phase air treatment: room air is the most likely vector and therefore a key element in the stopping of transmission of infection. (Figure 8). Although the transmission of SARS-CoV-2 infection occurs mainly through droplets that fall relatively rapidly to the ground, aerosol transmission is also possible and in dental office quite likely to occur. Small aerosolized particles smaller than $5 \mu \mathrm{m}$ contaminate the air and can levitate indoors where the air is not exchanged and disinfected for several hours. In addition to PPE, aerosol extractors and other elements preventing significant air contamination in the clinic, it is therefore appropriate to include other forms of air conditioning, in our case hooded UVC emitters combined with diffusers of biocidal oils, suitable for combination with UVC sterilization.

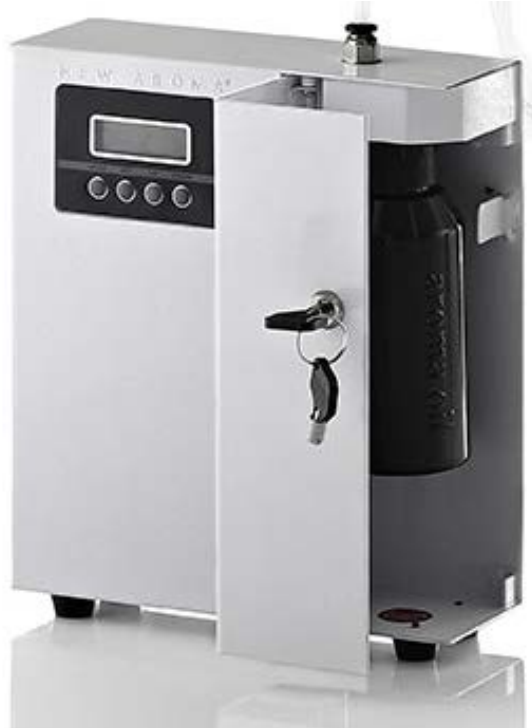

(a)

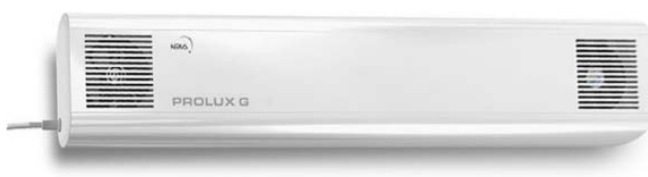

(b)

Figure 8. Key part of this biosafety protocol is combination of UVC emitters combined with diffusers of biocidal oils; (a) certified mini diffuser/nebulizer for virucidal aromatic oils for air-conditioning installation (up to1000 $\mathrm{m}^{3}$ ) New Aroma (b) certified hooded UVC Germicidal radiator PROLUX G30W A / SPH01, wall mounted 
Two hardware key elements are:

A) Germicidal radiator PROLUX G30W A / SPH01

- UVC lamp life of 8,000 hours

- for two-shift operation endurance 550 days $(2 \times 7.5 \mathrm{~h})$

- 1 emitter cycle is sufficient to sterilize a volume of $5.5 \mathrm{~m} \times 4 \mathrm{~m} \times 3.0 \mathrm{~m}$

Sterilization of the entire air volume of one separate room in clinic will therefore take approximately 30 minutes (time intervals between patients). SARS-CoV-2 virus particles are rapidly inactivated by UVC radiation.

B) Aroma Pro Mini - professional diffuser (aroma atomizer)

- For air conditioning

- Capacity up to $1000 \mathrm{~m}^{3}$ (www.NewAroma.sk accessed $15^{\text {th }}$ December 2021)

- Possibility to choose from several certified disinfectant oils.

- The disinfectant is present in the mixture in 3 weight percent at an emission of $5 \mathrm{ml}$ per hour (adjustable) to form an invisible aerosol dispersion in the air.

10. FFP3 shield active respirator is a special medical device with active FFP3 shield respirator - BioVYZR - Toronto USA based Vyzr Technologies. This device covers the wearer's face and protects against droplets and pathogens. It is defined as Powered Air Purifying Respirator (PAPR), a device typically used only in industrial and healthcare settings. This active respirator is not intended to be used regularly in this biosafety protocol. The purpose of this "last resort" is eventuality of a sudden appearance of a new mutant with gained new dangerous biological functions - possible high lethality, neuropathological affinity or onco-inductive attributes, where care of urgent patients shall remain uninterrupted.

With replaceable inlet and outlet FFP3 filters, with active ventilation with adjustable intensity, non-fogging shield, rechargeable battery. Suitable for long-term workload with frequent communication with the patient. It minimizes the risk of infection by healthcare professionals and thus contributes to increasing patient safety. However, it also works the other way around, protecting the patient from the healthcare provider thanks to the FFP3 output filter (Figure 9). To facilitate communication, we have purchased "voice amplifiers", devices commonly used by guides in museums. Such a device will make communication towards patients easier. Weight of the whole suit is approximately $2.3 \mathrm{~kg}$ with battery and amplifier, endurance 8+ hours, neoprene material, 3 sizes. 


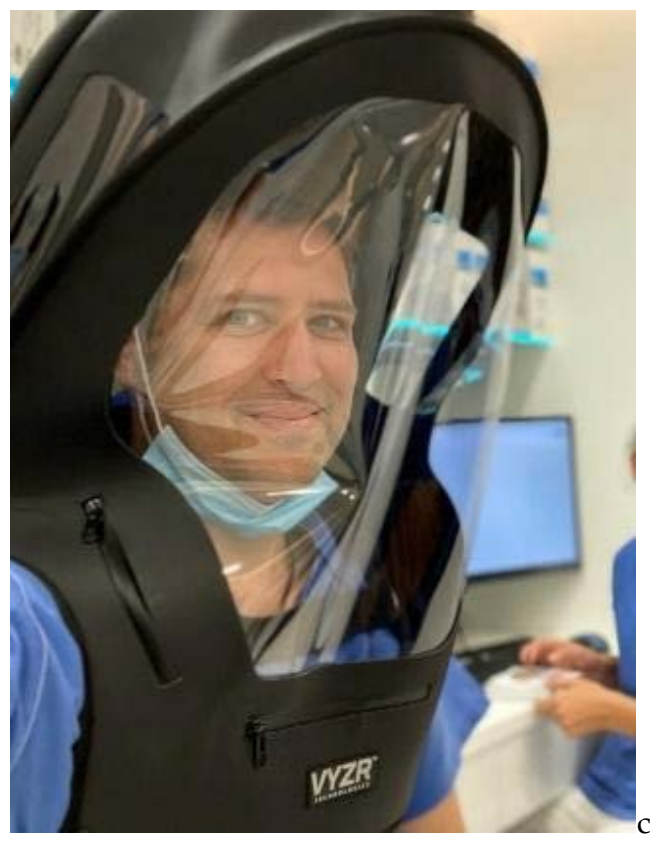

Figure 9. FFP3 active respirator is a special medical device - BioVYZR - Toronto USA based Vyzr Technologies. The purpose of this "last resort" suit is eventuality of a sudden appearance of a new strain with gained new dangerous biological properties - possibly high lethality, where care for urgent patients may remain uninterrupted.

\subsubsection{Other recommendations and patient management}

1. The scope of the measures specified in the protocol is adapted according to the type of procedure and the current epidemiological situation of SARS-CoV-2 infection for the given area.

2. The patient is ordered exclusively by phone or electronically

3. Before the visit, the patient has the option of a video consultation (Face time WhatsApp)

4. Before his physical visit, the patient receives a smart on-line anamnestic form, which also contains parts that make a $4 \mathrm{D}$ record of smile dynamics and others

5. The specific time, date and place of the consultation, diagnosis or treatment are sent as an electronic Google Calendar event after a telephone appointment with the patient and are confirmed to the patient's email. In this way, the patient does not meet unnecessarily with other patients in the waiting room

6. In the evening, before the date of the appointment, the nurse / dental assistant calls the patient and, in addition to reminding the appointment, finds out about his health condition, as sowing herpes, cough, muscle aches, fatigue, loss of smell or similar manifestations would delay the appointment and its physical appearance. clinic is unnecessary in this case.

7. During the physical visit to the clinic, before entering the outpatient clinic, the patient is examined with a non-contact thermometer, 4 samples of various scents for anosmia and signs an affidavit stating that no one is in quarantine or in its vicinity.

8. Before the patient enters the ambulance, it is completely sterilized after a previous patient covered with a Prolux 30W germicidal UVC emitter for 30-35 minutes, which is calculated to be sufficient to sterilize the whole unit. The air is also contaminated with a dispersion of certified biocidal disinfectant oil droplets by a professional diffuser NewAroma. At the same time, after the previous patient, all surfaces of the ambulance with which he could come into direct or indirect contact were disinfected. A 
30-minute ambulance sterilization protocol prevents each patient from being treated and requires simultaneous rotation of two to three separate compartments.

9. During the entire stay the medical staff moves around in protective equipment, as standard FFP3 respirators can be substituted with FFP2 respirators in periods with lower infection pressure. During outbreaks of unknown mutants, the active FFP3 shield respirators (Biovyzr) shall be used, all surfaces with which the health personnel came into contact is disinfected. Medical staff minimizes the time of mutual exposure - they do not share the same room during the service, they avoid eating together, changing clothes and eating any food or fluids in protected areas, they wear a standard three-layer face mask when in contact and regularly tested for COVID19.

10. The patient enters the clinic with the FFP2 on and puts his personal belongings in one specific place; then disinfects his hands with a contactless hand disinfectant dispenser - Germstar.

11. During the treatment in the mouth, the patient has a retractor fitted with the described special sterilizable aerosol aspirator connected to a surgical aspirator. This significantly reduces the aerosol dispersion formed during the removal of attachments or dental hygiene procedures.

The rooms were equipped with dental units Kavo Estetica E50 Life COMPACTchair machines, are subject of regular automatic disinfection of the internal water circulation in DVGW machines with a kit for intensive disinfection.

\subsection{Protocol development and evaluation}

The protocol has been in development since $8^{\text {th }}$ of February 2020 until $1^{\text {st }}$ of June 2020 (5 months).

The unchanged protocol in place was from $1^{\text {st }}$ of June 2020 until $30^{\text {th }}$ of November (18 months).

The protocol evaluation with auto-locked smart on-line forms was performed between $1^{\text {st }}$ of December and $12^{\text {th }}$ of December.

URL of the English evaluation form (smart Typeform form): https://sangreazul.typeform.com/BioSafety-ENG (accessed 15th December 2021)

\subsection{Protocol strengths and weaknesses}

What makes this protocol special:

- low cost, affordable, effective, sustainable

- does not require specific rebuilding of the current dental set-ups

- dimensioned for more infectious strains like Omicron

- not dependable on vaccination status or unreliable testing results

Weaknesses:

- Hard to clinically evaluate

- Possible overkill

- Unknown performance under Omicron 


\section{Results}

\subsection{Descriptive results}

From approximately 160 relevant patients, the smart online form has been filled out and submitted 115 times (Figure 2). 8 responses in English language the rest is in Slovak. The special fingerprint feature identified 3 duplicate answers that were removed. One of the responses was probably intentionally invalid as it repeatedly referred triple vaccination even before $3^{\text {rd }}$ shots were available in EU as well references events out of the observed 18-month period and entered various other oxymorons. This response has been evaluated as invalid. Final result is 111 responses.

All answers are in the table that as available online in public repository.

Approximately half of the responding patients were exposed to an infected person with Covid-19 (Figure 10). Exactly from 111 patients were 56 (50,5\%) directly exposed to SARS-Co-2 infection and 55 (49,5\%) were not (during the last 18-months).

Regarding dates of these exposures (Figure 11), they correlate with Slovak regional pandemic situation and is obvious difference between previous and the current wave.

$90(81 \%)$ of responding patients were vaccinated (Figure 12 and Table 1$)$, however, only 14 had Pfizer booster shot.

58 were vaccinated with 2 shots of Pfizer, 7 with Moderna, 4 with Janssen and 8 with AstraZeneca.

$45(50 \%)$ of all vaccinated received their last shot until June 2021, so with over 6 months since last vaccination their immunity from vaccines against Omicron variant will not be probably relevant.

The evaluation of the protocol reveals 9 patients reporting possibility of them being infectious during their visit at the clinic. There was no incident of personnel getting infected from patient or within work. Only two of the personnel got infected with covid in the past. Both cases were from well-known family sources.

38 responders got infected with the SARS.CoV.2 virus. 37 rejected possibility they got infected at the clinic under evaluated protocol. Only one of them considered this as possibility with lowest offered level of confidence.

There were 5 levels of confidence of this parameter 1-5:

$$
\begin{aligned}
& 1 \text { - Certainly not! } 0 \% \\
& 2-\mathbf{2 5 \%} \\
& 3 \text { - maybe } 50 \% \\
& 4 \text { - } 75 \% \\
& 5 \text { - Sure I've got it there } 100 \%
\end{aligned}
$$

In regard to question for biosafety feeling of patient during the dental procedure, (Figure 13) the feeling of patients was evaluated on the scale 1-5, where the 1 represents "no safety feeling “to 5 „,very safe “. From 111 patients mean feeling was M=4.59, SD= 0.732 .

37 patients answered the question "Could you have ever visited our clinic as already infected? ", (Figure 14) there was scale, which divided the risk of infectious status to 1 no, there were no chance to be infectious during the visit, until 5 - yes, I was infectious during the visit. $\mathrm{M}=1.32, \mathrm{SD}=.818$. All the infectious patients visiting the clinic were asymptomatic as they would not get through initial entry filter (Figure15). 


\subsection{Graphical interpretation of clinical evaluation of the protocol}

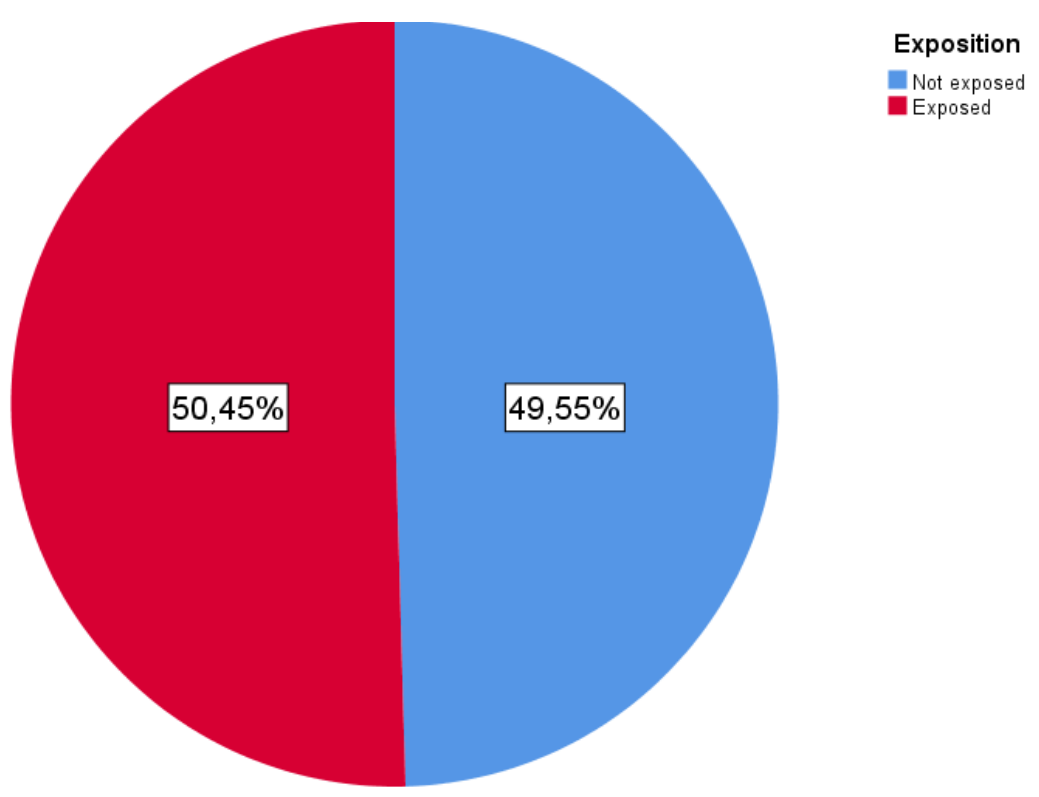

Figure 10. This graph represents distribution of exposed vs non exposed patients to someone diagnosed with Covid-19 during the last 18 months.

From 111 patients were 56 (50,5\%) exposed to SARS-Co-2 infection and $55(49,5 \%)$ were not (Figure 10). The incidence of the exposures to an infected person is in Figure 11.

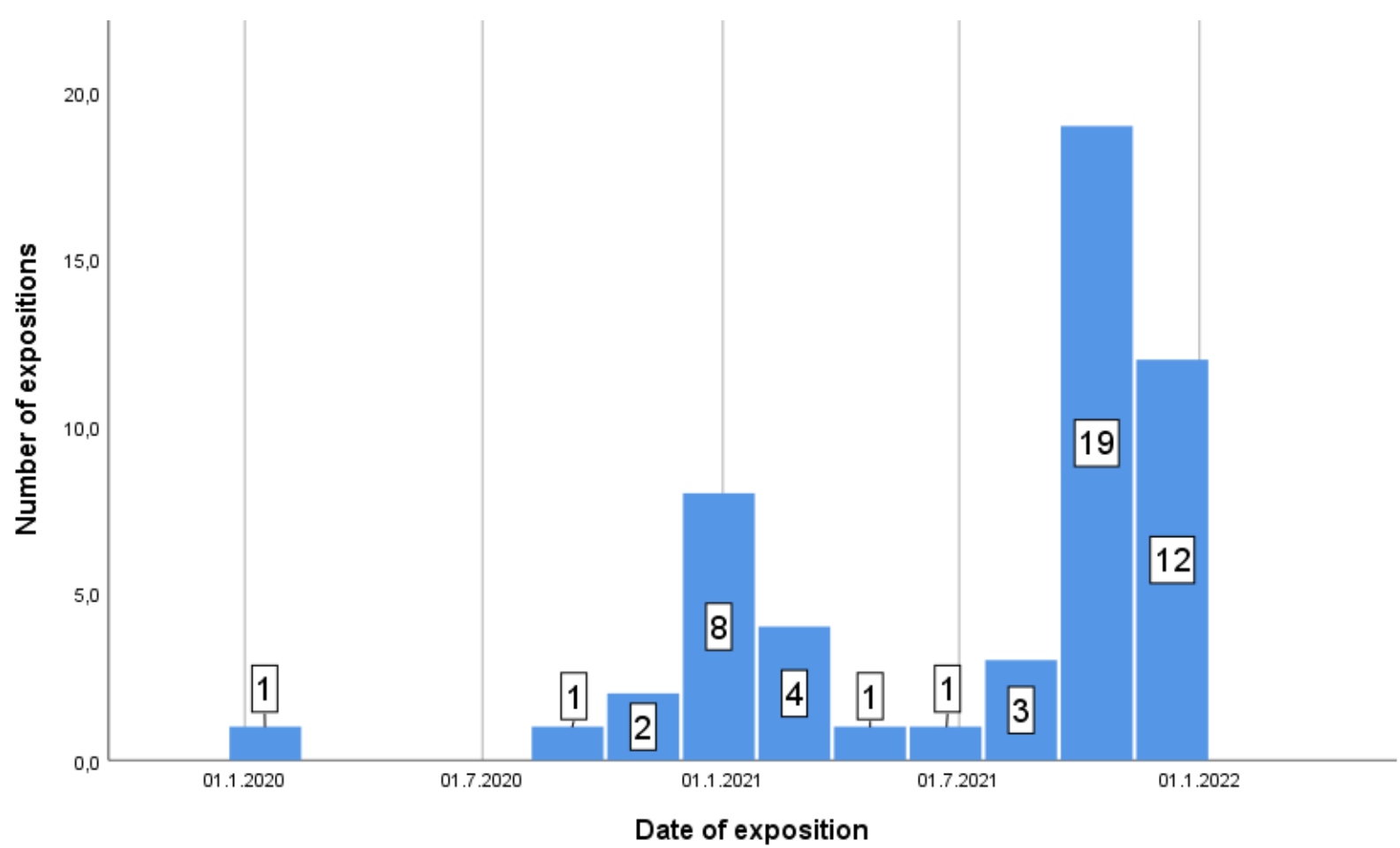

Figure 11. Frequency of the exposure to an infected person by the date. 


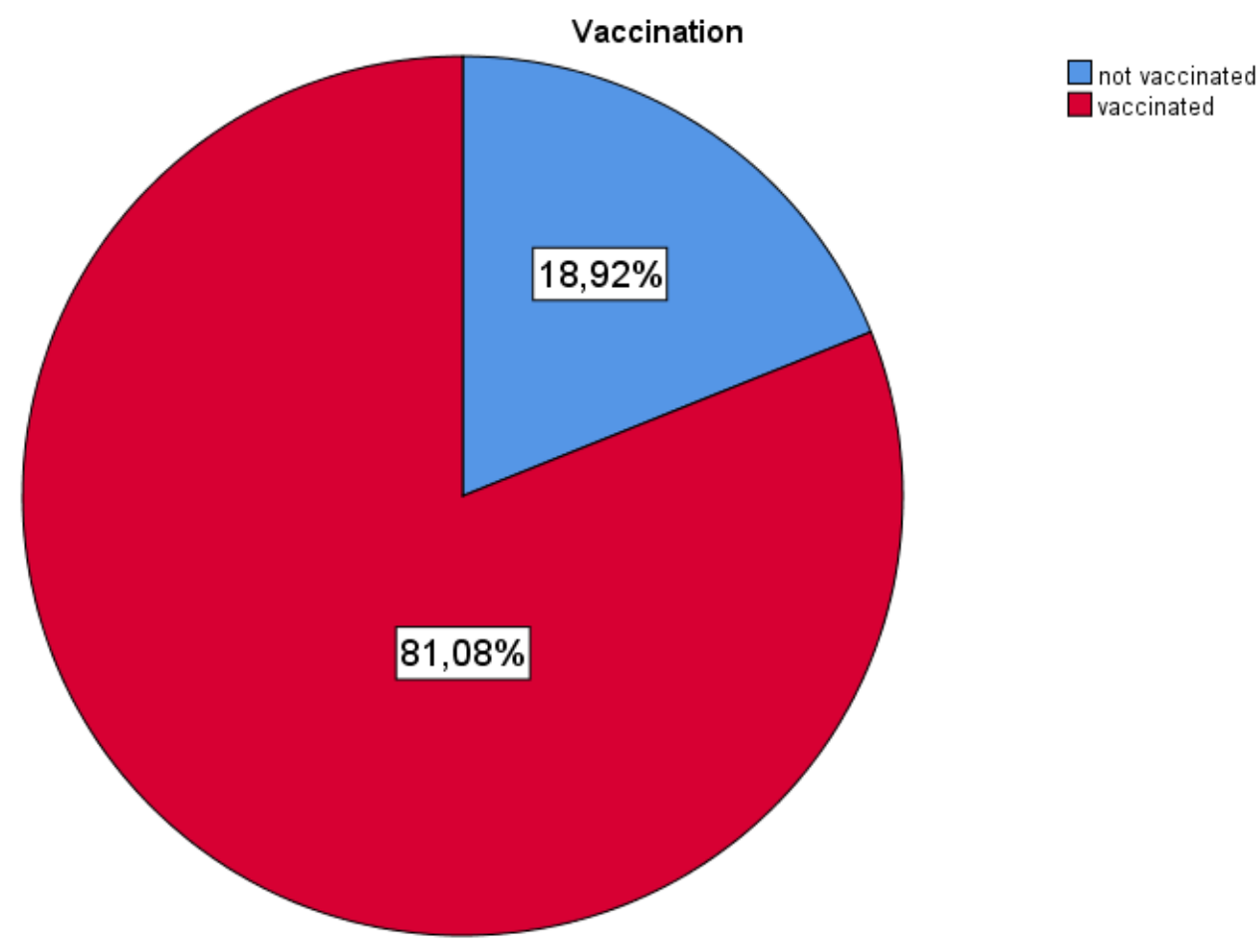

Figure 12. Vaccination distribution in patients

Table 1. Vaccination status of all responses

\begin{tabular}{ccc}
\hline Status & Frequency & Percent \\
\hline not vaccinated & 21 & 18,9 \\
vaccinated & 90 & 81,1 \\
Total & 111 & 100,0 \\
\hline
\end{tabular}

One-sample t-test revealed the significant difference in distribution of the exposures $\mathrm{t}(51)=7103,185, \mathrm{p}<0.001$ during the time. $\quad(\mathrm{M}=14.7 .2021 ; \mathrm{SD}=162 \mathrm{~d})$.

Independent sample $t$ test showed the significant higher number of exposures in the unvaccinated group $(\mathrm{N}=11, \mathrm{M}=3.91, \mathrm{SD}=1.3)$ and vaccinated group $(\mathrm{N}=45 ; \mathrm{M}=2.42, \mathrm{SD}=$ 1.438) t (54) $=3.127, \mathrm{p}=.003$.

There was no significant and low Pearson 's correlation (almost significant) between the date of exposition and number of the protentional contacts with SARS-Co-2 $\mathrm{r}=0.265$, $\mathrm{N}=52, \mathrm{p}=0.058$.

\section{Biosafety protocol}

The records of safety feeling of patients was on the scale 1-5, where the 1 represents „,no safety feeling "to 5 „,very safe “, from 111 patients mean feeling was M=4.59, SD= 0.732 (Figure 13, and Table 2). 


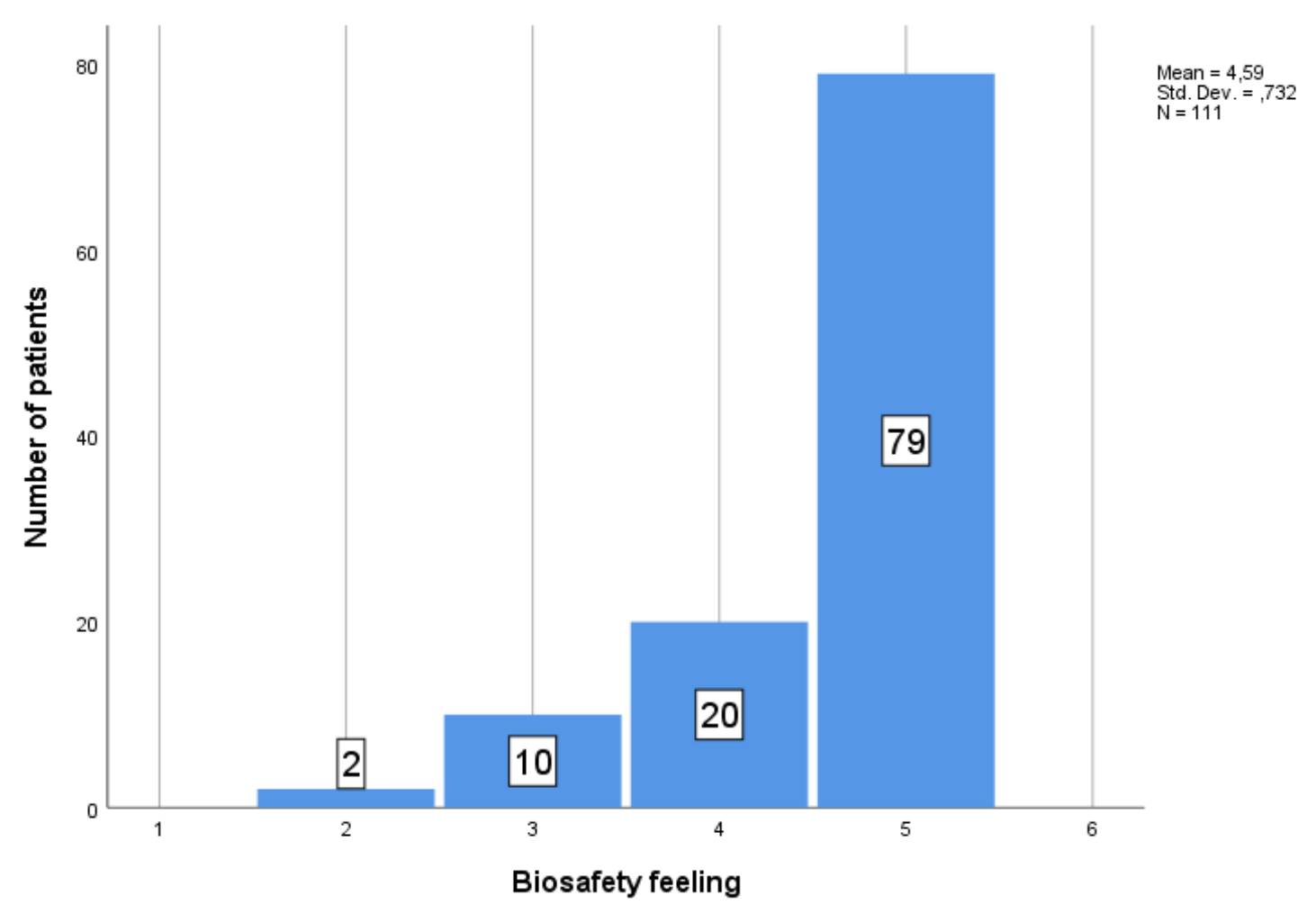

Figure 13. Number of the biosafety feeling records by value

Table 2. Biosafety feeling of patient during dental procedures

\begin{tabular}{|c|c|c|c|c|c|c|c|c|}
\hline & \multirow[b]{2}{*}{$\mathbf{N}$} & \multirow[b]{2}{*}{ Mean } & \multirow[b]{2}{*}{$\begin{array}{c}\text { Std. } \\
\text { Deviation }\end{array}$} & \multirow[b]{2}{*}{$\begin{array}{l}\text { Std. } \\
\text { Error }\end{array}$} & \multicolumn{2}{|c|}{$\begin{array}{c}95 \% \text { Confidence Interval } \\
\text { for Mean }\end{array}$} & \multirow[b]{2}{*}{ Minimum } & \multirow[b]{2}{*}{ Maximun } \\
\hline & & & & & $\begin{array}{l}\text { Lower } \\
\text { Bound }\end{array}$ & $\begin{array}{l}\text { Upper } \\
\text { Bound }\end{array}$ & & \\
\hline disease free & 74 & 4,55 & ,743 & , 086 & 4,38 & 4,73 & 2 & 5 \\
\hline $\mathrm{Ab}+$ & 3 & 4,67 & ,577 & 333 & 3,23 & 6,10 & 4 & 5 \\
\hline PCR/Ag + & 30 & 4,73 &, 583 & 106 & 4,52 & 4,95 & 3 & 5 \\
\hline multiple & 3 & 3,67 & 1,528 & ,882 &,- 13 & 7,46 & 2 & 5 \\
\hline other & 1 & 5,00 & & & & & 5 & 5 \\
\hline Total & 111 & 4,59 & ,732 & ,069 & 4,45 & 4,72 & 2 & 5 \\
\hline
\end{tabular}

Between the groups of unvaccinated patients, which one was declared as "Never got the vaccine against SARS-Co-2" $(\mathrm{N}=5, \mathrm{M}=5, \mathrm{SD}=0)$ and the second group was "Unvaccinated, but still waiting" $(\mathrm{N}=16, \mathrm{M}=4.5, \mathrm{SD}=.730)$, there was no significant difference between the biosafety feeling $\mathrm{t}(19)=-1.504, \mathrm{p}=.149$

Also, there was no statistical difference in biosafety feeling between in the group of vaccinated patients between the patients without booster dose $(\mathrm{N}=78, \mathrm{M}=4.56, \mathrm{SD}=.783)$ and with booster dose $(\mathrm{N}=12, \mathrm{M}=4.67, \mathrm{SD}=.492)$.

37 patients answered the question „Could you have ever visited our clinic as already infected? ", there was scale, which divided the risk of infectious status to 1 no, there were no chance to be infectious during the visit, until 5 - yes, I was infectious during the visit. $\mathrm{M}=1.32, \mathrm{SD}=.818$. 


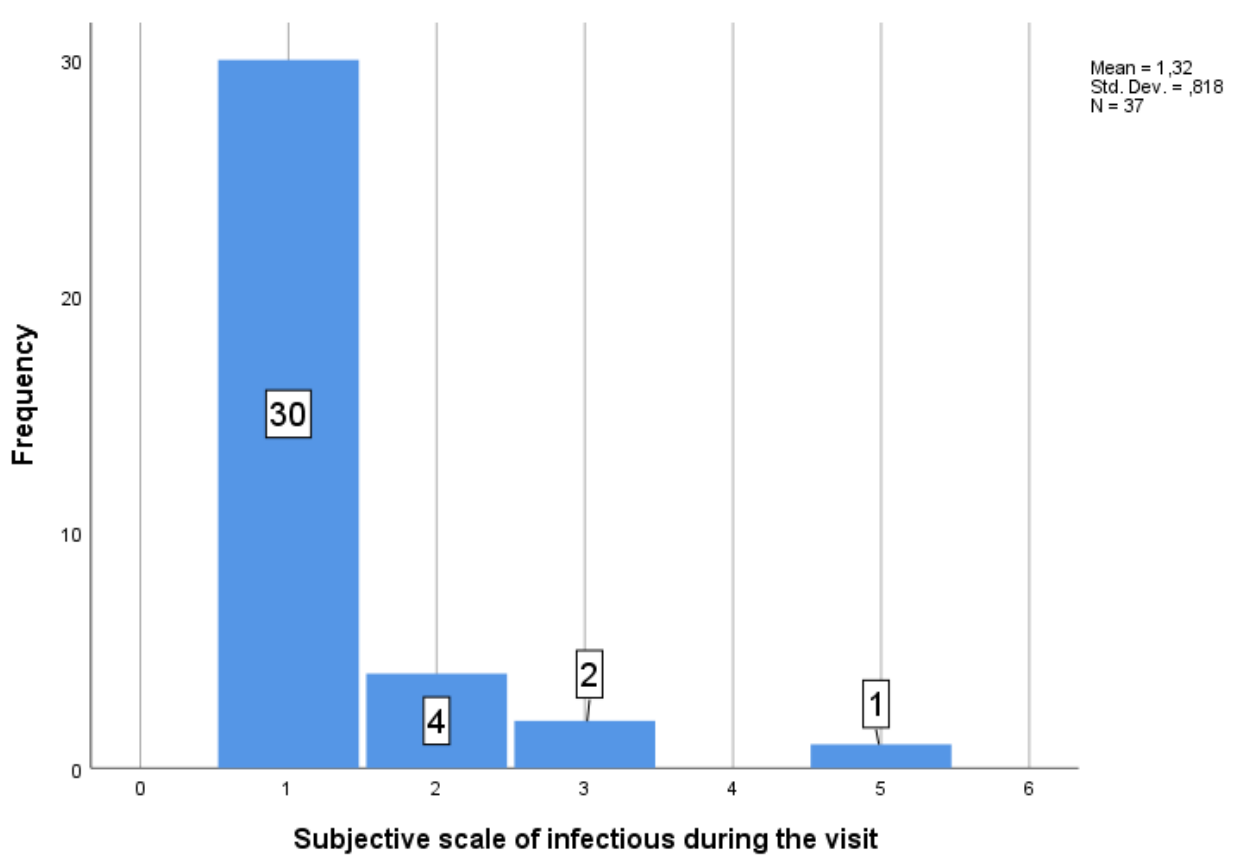

Figure 14. Number of the subjective infectious status during visit

For the patients, who answered the question of possible infectious status during the visit $(\mathrm{N}=6)$ (scale of potential risk was higher than 1$)$, they recorded the most no symptoms during the visit $\mathrm{N}=3(50 \%)$, headache $\mathrm{N}=2(33,3 \%)$ and pain of joints $\mathrm{N}=1(16,67 \%)$ (Figures 14 and 15).

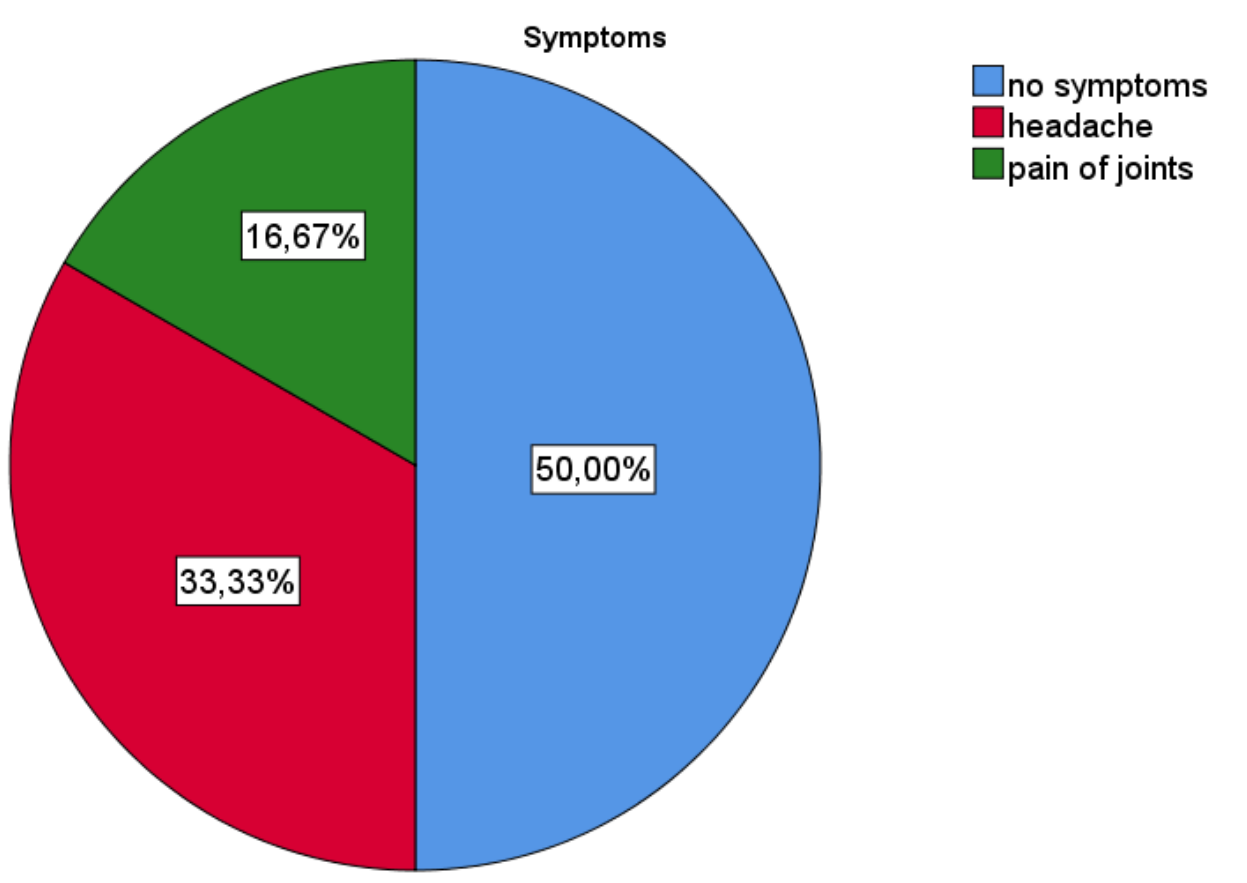

Figure 15. Potential infectious patients during the visit and spectrum of their symptoms

There was medium correlation between number of exposures and potentially infectious patients $\mathrm{r}(28)=.402 ; \mathrm{p}=.034$. 


\section{Discussion}

In this article authors have presented a biosafety protocol with further context to orthodontic care facing Omicron variant with new epidemiological properties. With this new variant we anticipate a higher transmissibility and lower protection from vaccines, albeit with milder clinical symptoms $[76-82,84,85,87,88]$. So, there is more likely to have an asymptomatic carrier on dental procedure in a near future than is today.

The results of the survey have demonstrated, that patient entry-symptomatic-screening prevented symptomatic patients present in the dental procedures. Survey also revealed that most of the vaccinated patients have probably very low or nearly none protection from vaccines $[37,43,74,76,78]$.

The weakness of this study is the clinical evaluation of clinical performance of the true safety of this biosafety protocol. On-line survey does not represent representative sample, it depends on the self-assessment of the responders. As well there is a high probability that responders are more responsible part of the targeted group.

The results can be interpreted from the perspective of previous studies presented in the Introduction chapter as supporting the validity of the protocol, albeit not proving him safe rather proving him not unsafe. Wider and multicentric research is necessary to prove reliability of this settings. Ideally with similar clinic not following the protocol, however working in the similar geographical location and intensity.

Approximately half of the responding patients were exposed to an infected person with Covid-19 however this is self-assessment evaluation. It is interesting that dates of these exposures correlate with Slovak regional pandemic situation in those times. It is also is obvious difference between previous and the current wave. The frequency of exposures during the current wave is higher than in the previous when more strict lockdowns were implied. $50 \%$ of all vaccinated patients have received their last shot before June 2021, so with over 6 months since the last vaccination their immunity from vaccines against Omicron variant will not be probably relevant $[37,78]$.

More transmissible Omicron variant defines a new chapter of COVID-19 pandemics. As it is spreading at a rate we have not seen with any previous variants, there is a concern that people are dismissing omicron as mild, not learning from the recent past. Even if omicron does cause less severe disease, the sheer number of cases could once again overwhelm unprepared health systems [124].

Omicron contains mutations associated with higher levels of immune escape, higher transmissibility and an improved ability to bind to cells. But there are also many mutations within the new variant that are not yet understood. As we have no idea what these new mutations do yet, it is logical to stay cautious and responsible [124].

Predictions of practicality and efficiency of this prospective setting for dental care are difficult, as they face only the preliminary findings about Omicron variant and society attitudes are changing. We are currently facing considerations of Omicron as a possible pandemic-ender, with some people including some politicians willing to take the risk for the sake of economy. Some people are willing to even voluntarily get infected despite the Omicrons's c. The known risks of the infection with impact on the nervous system, heart tissue or the risk of long covid are known. It stays yet unclear whether Omicron will have any of the "silent" effects seen with earlier variants, such as self-attacking antibodies, sperm impairments and changes in insulin-producing cells.

Only time will reveal if there were other hidden risks for our health. In this regard, only recently researchers have found strong evidence that it's an infection with the Epstein-Barr virus - a particularly ubiquitous member of the herpesvirus family, best known for causing mononucleosis, triggers multiple sclerosis (MS). Infection with Epstein-Barr increased the likelihood of developing multiple sclerosis, by more than 32-fold [125].

However, as this new era puts current vaccines into different perspective, masks, ventilation and hygiene stay again unaffected. Vaccines are tools that have the greatest impact when they are used to protect those who are most at risk. They are the last line of our defence. Vaccines cannot be today considered as substitute for masks, distancing, 
ventilation or hand hygiene. It also seems logical that Omicron-like strains are here to stay. It can be fought with measures that work today and that must be sustainable. This presented biosafety protocol addresses higher risks suggested by preliminary observations that indicate Omicron spreads faster and escapes antibodies more readily than previous variants. Loss of smell and taste is clinically frequent in older variants, now with Omicron, Sore throat and night sweats are reported frequently. The protocol anticipates the increase of reinfections and cases of mild breakthrough infections in people who are vaccinated is highly probable [87].

\section{Conclusions}

During 18 months of this biosafety protocol in place, no proven infection of healthcare personnel or patient occurred in the dental clinic despite treatment of at least nine high-probability infectious patients undertaking dental procedures. While more infectious Omicron does appear to be clinically less severe compared to Delta, its long-term effects are yet unknown and dental office must not risk patients` infection [126].

Supplementary Materials: The following are available online at https://docs.google.com/spreadsheets/d/1lznYWB32gTb282v4HJbQRY Uw42xXnBhNj-vnmvJvKE/edit?usp=sharing

Author Contributions: are defined during the online submission process.

Funding: This research was funded by the KEGA grant agency of the Ministry of Education, Science, Research, and Sport of the Slovak Republic (Grant No. 081UK-4/2021); Data analysis was supported by the grants APVV: SK-BY-RD-19-0019 and KEGA: 041UK-4/2020.

Institutional Review Board Statement: The study was conducted according to the guidelines of the Declaration of Helsinki, and no approval was necessary by the Ethics Committee. Ethical review and approval were waived for this study, due to the fact that no experimental materials or approaches were used. All used materials and machines were fully certified and are still available on the market.

Informed Consent Statement: Written informed consent was obtained from all subjects involved in the study.

Data Availability Statement: We fully adhere to Data Availability Statements in section "MDPI Research Data Policies" at https://www.mdpi.com/ethics.

Acknowledgments: We acknowledge support of Medical faculty Comenius University in Bratislava, technological support of digital Dental lab infrastructure of 3Dent Medical s.r.o company as well as dental clinic Sangre Azul s.r.o.

Conflicts of Interest: The authors declare no conflict of interest.

\section{References}

1. Cummins, C.P.; Ajayi, O.J.; Mehendale, F. v.; Gabl, R.; Viola, I.M. The Dispersion of Spherical Droplets in SourceSink Flows and Their Relevance to the COVID-19 Pandemic. Physics of Fluids 2020, 32, 083302, doi:10.1063/5.0021427.

2. Javaid, M.; Haleem, A.; Singh, R.P.; Suman, R. Dentistry 4.0 Technologies Applications for Dentistry during COVID-19 Pandemic. Sustainable Operations and Computers 2021, 2, 87-96, doi:10.1016/J.SUSOC.2021.05.002.

3. $\quad$ Pai, S.; Patili, V.; Kamath, R.; Mahendra, M.; Singhal, D.K.; Bhat, V. Work-Life Balance amongst Dental Professionals during the COVID-19 Pandemic-A Structural Equation Modelling Approach. PLoS ONE 2021, 16, doi:10.1371/JOURNAL.PONE.0256663.

4. Thurzo, A.; Kurilová, V.; Varga, I. Artificial Intelligence in Orthodontic Smart Application for Treatment Coaching and Its Impact on Clinical Performance of Patients Monitored with AI-TeleHealth System. Healthcare 2021, Vol. 9, Page 1695 2021, 9, 1695, doi:10.3390/HEALTHCARE9121695. 
5. Cabrera-Tasayco, F.D.P.; Rivera-Carhuavilca, J.M.; Atoche-Socola, K.J.; Peña-Soto, C.; Arriola-Guillén, L.E. Biosafety Measures at the Dental Office After the Appearance of COVID-19: A Systematic Review. Disaster Medicine and Public Health Preparedness 2020, 1-5, doi:10.1017/DMP.2020.269.

6. Abdelrahim, R.K.; Abdoun, H.A.E.; Koppolu, P.; Swapna, L.A. Infection Control Measures in Dental Clinics during Coronavirus Disease-19 Pandemic in Kingdom of Saudi Arabia: A Pilot Study. Open Access Macedonian Journal of Medical Sciences 2021, 9, 61-67, doi:10.3889/oamjms.2021.5898.

7. Campus, G.; Diaz-Betancourt, M.; Cagetti, M.G.; Carvalho, J.C.; Carvalho, T.S.; Cortés-Martinicorena, J.F.; Deschner, J.; Douglas, G.V.A.; Giacaman, R.; Machiulskiene, V.; et al. Study Protocol for an Online Questionnaire Survey on Symptoms/Signs, Protective Measures, Level of Awareness and Perception Regarding COVID-19 Outbreak among Dentists. A Global Survey. International Journal of Environmental Research and Public Health 2020, Vol. 17, Page 5598 2020, 17, 5598, doi:10.3390/IJERPH17155598.

8. Campus, G.; Diaz-Betancourt, M.; Cagetti, M.G.; Carvalho, J.C.; Carvalho, T.S.; Cortés-Martinicorena, J.F.; Deschner, J.; Douglas, G.V.A.; Giacaman, R.; Machiulskiene, V.; et al. Study Protocol for an Online Questionnaire Survey on Symptoms/Signs, Protective Measures, Level of Awareness and Perception Regarding COVID-19 Outbreak among Dentists. A Global Survey. International Journal of Environmental Research and Public Health 2020, Vol. 17, Page 5598 2020, 17, 5598, doi:10.3390/IJERPH17155598.

9. Shihabi, S.; Nesser, S. al; Hamadah, O.; Shihabi, S.; Nesser, S. al; Hamadah, O. The Preventive Measures Adopted during Dental Practice by the Dentists in a Low-Income Country to Prevent the Transmission of COVID-19: A Questionnaire-Based Survey. Indian Journal of Medical Sciences 2021, 73, 15-20, doi:10.25259/IJMS_231_2020.

10. Pan, Y.; Liu, H.; Chu, C.; Li, X.; Liu, S.; Lu, S. Transmission Routes of SARS-CoV-2 and Protective Measures in Dental Clinics during the COVID-19 Pandemic. American journal of dentistry 2020, 33, 129-134.

11. Moraes, R.R.; Correa, M.B.; Queiroz, A.B.; Daneris, Â.; Lopes, J.P.; Pereira-Cenci, T.; D'Avila, O.P.; Cenci, M.S.; Lima, G.S.; Demarco, F.F. COVID-19 Challenges to Dentistry in the New Pandemic Epicenter: Brazil. PLOS ONE 2020, 15, e0242251, doi:10.1371/journal.pone.0242251.

12. Siles-Garcia, A.A.; Alzamora-Cepeda, A.G.; Atoche-Socola, K.J.; Peña-Soto, C.; Arriola-Guillén, L.E. Biosafety for Dental Patients During Dentistry Care After COVID-19: A Review of the Literature. Disaster Medicine and Public Health Preparedness 2021, 15, e43-e48, doi:10.1017/DMP.2020.252.

13. Manuballa, S.; Abdelmaseh, M.; Tasgaonkar, N.; Frias, V.; Hess, M.; Crow, H.; Andreana, S.; Gupta, V.; Wooten, K.E.; Markiewicz, M.R.; et al. Managing the Oral Health of Cancer Patients During the COVID-19 Pandemic: Perspective of a Dental Clinic in a Cancer Center. Journal of Clinical Medicine 2020, Vol. 9, Page 3138 2020, 9, 3138, doi:10.3390/JCM9103138.

14. Meng, L.; Hua, F.; Bian, Z. Coronavirus Disease 2019 (COVID-19): Emerging and Future Challenges for Dental and Oral Medicine. Journal of dental research 2020, 99, 481-487, doi:10.1177/0022034520914246.

15. Froum, S.; Froum, S. Incidence of COVID-19 Virus Transmission in Three Dental Offices: A 6-Month Retrospective Study. The International journal of periodontics $\mathcal{E}$ restorative dentistry 2020, 40, 853-859, doi:10.11607/PRD.5455.

16. Hartig, M.; Stephens, C.; Foster, A.; Fontes, D.; Kinzel, M.; García-Godoy, F. Stopping the COVID-19 Pandemic in Dental Offices: A Review of SARS-CoV-2 Transmission and Cross-Infection Prevention. Experimental Biology and Medicine 2021, 246, 2381-2390, doi:10.1177/15353702211034164.

17. Peng, X.; Xu, X.; Li, Y.; Cheng, L.; Zhou, X.; Ren, B. Transmission Routes of 2019-NCoV and Controls in Dental Practice. International journal of oral science 2020, 12, doi:10.1038/S41368-020-0075-9. 
18. Araujo, M.W.B.; Estrich, C.G.; Mikkelsen, M.; Morrissey, R.; Harrison, B.; Geisinger, M.L.; Ioannidou, E.; Vujicic, M. COVID-2019 among Dentists in the United States: A 6-Month Longitudinal Report of Accumulative Prevalence and Incidence. Journal of the American Dental Association 2021, 152, 425-433, doi:10.1016/J.ADAJ.2021.03.021/ATTACHMENT/20D50BF3-538A-42E5-BA8B-1BCC3D8E5463/MMC1.DOCX.

19. Ge, Z. yu; Yang, L. ming; Xia, J. jia; Fu, X. hui; Zhang, Y. zhen Possible Aerosol Transmission of COVID-19 and Special Precautions in Dentistry. Journal of Zhejiang University. Science. B 2020, 21, 361, doi:10.1631/JZUS.B2010010.

20. Dabiri, D.; Conti, S.R.; Sadoughi Pour, N.; Chong, A.; Dadjoo, S.; Dabiri, D.; Wiese, C.; Badal, J.; Hoogland, M.A.; Conti, H.R.; et al. A Multi-Disciplinary Review on the Aerobiology of COVID-19 in Dental Settings. Frontiers in Dental Medicine 2021, 0, 66, doi:10.3389/FDMED.2021.726395.

21. Hamedani, S.; Farshidfar, N.; Ziaei, A.; Pakravan, H. The Dilemma of COVID-19 in Dental Practice Concerning the Role of Saliva in Transmission: A Brief Review of Current Evidence. European oral research 2020, 54, 92-100, doi:10.26650/EOR.20200050.

22. Meethil, A.P.; Saraswat, S.; Chaudhary, P.P.; Dabdoub, S.M.; Kumar, P.S. Sources of SARS-CoV-2 and Other Microorganisms in Dental Aerosols. Journal of Dental Research 2021, 100, 817-823, doi:10.1177/00220345211015948.

23. Sommerstein, R.; Fux, C.A.; Vuichard-Gysin, D.; Abbas, M.; Marschall, J.; Balmelli, C.; Troillet, N.; Harbarth, S.; Schlegel, M.; Widmer, A.; et al. Risk of SARS-CoV-2 Transmission by Aerosols, the Rational Use of Masks, and Protection of Healthcare Workers from COVID-19. Antimicrobial resistance and infection control 2020, 9, doi:10.1186/S13756-020-00763-0.

24. Meister, T.L.; Brüggemann, Y.; Todt, D.; Conzelmann, C.; Müller, J.A.; Groß, R.; Münch, J.; Krawczyk, A.; Steinmann, J.; Steinmann, J.; et al. Virucidal Efficacy of Different Oral Rinses Against Severe Acute Respiratory Syndrome Coronavirus 2. The Journal of Infectious Diseases 2020, 222, 1289-1292, doi:10.1093/infdis/jiaa471.

25. Perry, E. How I Chose a Preprocedural Rinse I Registered Dental Hygienists Available online: https://www.rdhmag.com/infection-control/article/14204889/how-i-chose-a-preprocedural-rinse (accessed on 15 December 2021).

26. Rutala, W.A.; Weber, D.J. Guideline for Disinfection and Sterilization in Healthcare Facilities, 2008.

27. Ortega, K.L.; Rech, B.O.; el Haje, G.L.C.; Gallo, C.B.; Pérez-Sayáns, M.; Braz-Silva, P.H. Do Hydrogen Peroxide Mouthwashes Have a Virucidal Effect? A Systematic Review. Journal of Hospital Infection 2020, 106, 657-662, doi:10.1016/J.JHIN.2020.10.003.

28. Don't Use Hydrogen Peroxide as a COVID-19 Pre-Procedural Rinse, Experts Say - Dental Tribune Canada Available online: https:/ca.dental-tribune.com/news/dont-use-hydrogen-peroxide-as-a-covid-19-preprocedural-rinse-experts-say/ (accessed on 15 December 2021).

29. Moskowitz, H.; Sci, M.M.-I.J.E.D.; 2020, undefined Comparative Analysis of Antiviral Efficacy of Four Different Mouthwashes against Severe Acute Respiratory Syndrome Coronavirus 2: An in Vitro Study. familysmilesfortlee.com, doi:10.5005/jp-journals-10029-1209.

30. Health Ontario, P. Rapid Review: Open Operatory Dental Setting Infection Control Practices and Risk of Transmission during Aerosol-Generating Dental Procedures 1 RAPID REVIEW.

31. Steinhauer, K.; Meister, T.L.; Todt, D.; Krawczyk, A.; Paßvogel, L.; Becker, B.; Paulmann, D.; Bischoff, B.; Pfaender, S.; Brill, F.H.H.; et al. Comparison of the In-Vitro Efficacy of Different Mouthwash Solutions Targeting SARS-CoV-2 Based on the European Standard EN 14476. The Journal of hospital infection 2021, 111, 180-183, doi:10.1016/J.JHIN.2021.01.031. 
32. Oliveira, M.M.M.; Almeida, A.C. de; Rodrigues, C.M. de C.; Sol, I.; Meneses-Santos, D. COVID-19 - Mouthwash in Dental Clinical Practice: Review. ARCHIVES OF HEALTH INVESTIGATION 2021, 10, 6-10, doi:10.21270/ARCHI.V10I1.5283.

33. Mohd-Said, S.; Mohd-Dom, T.N.; Suhaimi, N.; Rani, H.; McGrath, C. Effectiveness of Pre-Procedural Mouth Rinses in Reducing Aerosol Contamination During Periodontal Prophylaxis: A Systematic Review. Frontiers in Medicine 2021, 8, 632, doi:10.3389/FMED.2021.600769/BIBTEX.

34. Kelly, N.; Nic Íomhair, A.; McKenna, G. Can Oral Rinses Play a Role in Preventing Transmission of Covid 19 Infection? Evidence-Based Dentistry 2020 21:2 2020, 21, 42-43, doi:10.1038/s41432-020-0099-1.

35. Mohebbi, S.Z.; Ebrahimi, T.; Shamshiri, A.R. Do Mouthwashes Reduce Covid-19 Viral Load during Dental Procedures and Oropharyngeal Examinations? A Systematic Review. 2021, doi:10.20944/PREPRINTS202106.0249.V1.

36. Butera, A.; Maiorani, C.; Natoli, V.; Bruni, A.; Coscione, C.; Magliano, G.; Giacobbo, G.; Morelli, A.; Moressa, S.; Scribante, A. Bio-Inspired Systems in Nonsurgical Periodontal Therapy to Reduce Contaminated Aerosol during COVID-19: A Comprehensive and Bibliometric Review. Journal of Clinical Medicine 2020, Vol. 9, Page 3914 2020, 9, 3914, doi:10.3390/JCM9123914.

37. Farshidfar, N.; Jafarpour, D.; Hamedani, S.; Dziedzic, A.; Tanasiewicz, M. Proposal for Tier-Based Resumption of Dental Practice Determined by COVID-19 Rate, Testing and COVID-19 Vaccination: A Narrative Perspective. Journal of Clinical Medicine 2021, Vol. 10, Page 2116 2021, 10, 2116, doi:10.3390/JCM10102116.

38. Shirazi, S.; Stanford, C.M.; Cooper, L.F. Characteristics and Detection Rate of SARS-CoV-2 in Alternative Sites and Specimens Pertaining to Dental Practice: An Evidence Summary. Journal of Clinical Medicine 2021, Vol. 10, Page 1158 2021, 10, 1158, doi:10.3390/JCM10061158.

39. López-Verdín, S.; Prieto-Correa, J.R.; Molina-Frechero, N.; Bologna-Molina, R. Screening Test for COVID-19 in Dentai Practice: Best Options. American Journal of Dentistry 2021, 34, 127-131.

40. Jawad, H.; Hodson, N.A.; Nixon, P.J. A Review of Dental Treatment of Head and Neck Cancer Patients, before, during and after Radiotherapy: Part 1. British Dental Journal 2015, 218, 65-68, doi:10.1038/sj.bdj.2015.28.

41. Joshi, V.K. Dental Treatment Planning and Management for the Mouth Cancer Patient. Oral Oncology 2010, 46, 475-479, doi:10.1016/j.oraloncology.2010.03.010.

42. Bertuzzi, A.F.; Ciccarelli, M.; Marrari, A.; Gennaro, N.; Dipasquale, A.; Giordano, L.; Cariboni, U.; Quagliuolo, V.L.; Alloisio, M.; Santoro, A. Impact of Active Cancer on COVID-19 Survival: A Matched-Analysis on 557 Consecutive Patients at an Academic Hospital in Lombardy, Italy. British Journal of Cancer 2021, 125, 358-365, doi:10.1038/s41416-021-01396-9.

43. Herishanu, Y.; Avivi, I.; Aharon, A.; Shefer, G.; Levi, S.; Bronstein, Y.; Morales, M.; Ziv, T.; Shorer Arbel, Y.; Scarfò, L.; et al. Efficacy of the BNT162b2 MRNA COVID-19 Vaccine in Patients with Chronic Lymphocytic Leukemia. Blood 2021, 137, 3165-3173, doi:10.1182/blood.2021011568.

44. Zhang, S.; Guo, M.; Wu, F.; Xiong, N.; Ma, Y.; Wang, Z.; Duan, L.; Chen, L.; Ouyang, H.; Jin, Y. Factors Associated with Asymptomatic Infection in Health-Care Workers with Severe Acute Respiratory Syndrome Coronavirus 2 Infection in Wuhan, China: A Multicentre Retrospective Cohort Study. Clinical Microbiology and Infection 2020, 26, 1670-1675, doi:10.1016/j.cmi.2020.08.038.

45. Sinjari, B.; D’ardes, D.; Santilli, M.; Rexhepi, I.; D'addazio, G.; Carlo, P. di; Chiacchiaretta, P.; Caputi, S.; Cipollone, F. SARS-CoV-2 and Oral Manifestation: An Observational, Human Study. Journal of Clinical Medicine 2020, Vol. 9, Page 3218 2020, 9, 3218, doi:10.3390/JCM9103218. 
46. Amorim Dos Santos, J.; Normando, A.G.C.; Carvalho da Silva, R.L.; Acevedo, A.C.; de Luca Canto, G.; Sugaya, N.; Santos-Silva, A.R.; Guerra, E.N.S. Oral Manifestations in Patients with COVID-19: A 6-Month Update. Journal of dental research 2021, 100, 1321-1329, doi:10.1177/00220345211029637.

47. Aragoneses, J.; Suárez, A.; Algar, J.; Rodríguez, C.; López-Valverde, N.; Aragoneses, J.M. Oral Manifestations of COVID-19: Updated Systematic Review With Meta-Analysis. Frontiers in Medicine 2021, 8, 1423, doi:10.3389/FMED.2021.726753/BIBTEX.

48. Orilisi, G.; Mascitti, M.; Togni, L.; Monterubbianesi, R.; Tosco, V.; Vitiello, F.; Santarelli, A.; Putignano, A.; Orsini, G. Oral Manifestations of COVID-19 in Hospitalized Patients: A Systematic Review. International Journal of Environmental Research and Public Health 2021, Vol. 18, Page 12511 2021, 18, 12511, doi:10.3390/IJERPH182312511.

49. Hocková, B.; Riad, A.; Valky, J.; Šulajová, Z.; Stebel, A.; Slávik, R.; Bečková, Z.; Pokorná, A.; Klugarová, J.; Klugar, M. Oral Complications of ICU Patients with COVID-19: Case-Series and Review of Two Hundred Ten Cases. Journal of Clinical Medicine 2021, Vol. 10, Page 581 2021, 10, 581, doi:10.3390/JCM10040581.

50. Coke, C.J.; Davison, B.; Fields, N.; Fletcher, J.; Rollings, J.; Roberson, L.; Challagundla, K.B.; Sampath, C.; Cade, J.; Farmer-Dixon, C.; et al. SARS-CoV-2 Infection and Oral Health: Therapeutic Opportunities and Challenges. Journal of Clinical Medicine 2021, Vol. 10, Page 156 2021, 10, 156, doi:10.3390/JCM10010156.

51. Mekhemar, M.; Attia, S.; Dörfer, C.; Conrad, J. The Psychological Impact of the COVID-19 Pandemic on Dentists in Germany. Journal of Clinical Medicine 2021, Vol. 10, Page 1008 2021, 10, 1008, doi:10.3390/JCM10051008.

52. Pylińska-Dąbrowska, D.; Starzyńska, A.; Jerzy Cubała, W.; Ragin, K.; Alterio, D.; Jereczek-Fossa, B.A. Psychological Functioning of Patients Undergoing Oral Surgery Procedures during the Regime Related with SARS-CoV-2 Pandemic. Journal of Clinical Medicine 2020, Vol. 9, Page 3344 2020, 9, 3344, doi:10.3390/JCM9103344.

53. Emodi-Perlman, A.; Eli, I.; Smardz, J.; Uziel, N.; Wieckiewicz, G.; Gilon, E.; Grychowska, N.; Wieckiewicz, M. Temporomandibular Disorders and Bruxism Outbreak as a Possible Factor of Orofacial Pain Worsening during the COVID-19 Pandemic-Concomitant Research in Two Countries. Journal of Clinical Medicine 2020, Vol. 9, Page 3250 2020, 9, 3250, doi:10.3390/JCM9103250.

54. Olszewska, A.; Rzymski, P. Children's Dental Anxiety during the COVID-19 Pandemic: Polish Experience. Journal of Clinical Medicine 2020, Vol. 9, Page 2751 2020, 9, 2751, doi:10.3390/JCM9092751.

55. Ahmadi, H.; Ebrahimi, A.; Ghorbani, F. The Impact of COVID-19 Pandemic on Dental Practice in Iran: A Questionnaire-Based Report. BMC Oral Health 2020, 20, 1-9, doi:10.1186/S12903-020-01341-X/TABLES/6.

56. Faccini, M.; Ferruzzi, F.; Mori, A.A.; Santin, G.C.; Oliveira, R.C.; Oliveira, R.C.G. de; Queiroz, P.M.; Salmeron, S.; Pini, N.I.P.; Sundfeld, D.; et al. Dental Care during COVID-19 Outbreak: A Web-Based Survey. European Journal of Dentistry 2020, 14, S14-S19, doi:10.1055/s-0040-1715990.

57. Derruau, S.; Bouchet, J.; Nassif, A.; Baudet, A.; Yasukawa, K.; Lorimier, S.; Prêcheur, I.; Bloch-Zupan, A.; Pellat, B.; Chardin, H.; et al. COVID-19 and Dentistry in 72 Questions: An Overview of the Literature. Journal of Clinical Medicine 2021, Vol. 10, Page 779 2021, 10, 779, doi:10.3390/JCM10040779.

58. Cagetti, M.G.; Cairoli, J.L.; Senna, A.; Campus, G. COVID-19 Outbreak in North Italy: An Overview on Dentistry. A Questionnaire Survey. International Journal of Environmental Research and Public Health 2020, Vol. 17, Page 3835 2020, 17, 3835, doi:10.3390/IJERPH17113835.

59. SFIKAS, P.M. Teledentistry: Legal and Regulatory Issues Explored. The Journal of the American Dental Association 1997, 128, 1716-1718, doi:10.14219/JADA.ARCHIVE.1997.0137.

60. Kravitz, N.; Burris, B.; Butler, D.; Dabney, C. Teledentistry, Do-It-Yourself Orthodontics, and Remote Treatment Monitoring. Journal of clinical orthodontics : JCO 2016, 50, 718-726. 
61. Park, J.H.; Rogowski, L.; Kim, J.H.; al Shami, S.; Howell, S.E.I. Teledentistry Platforms for Orthodontics. Journal of Clinical Pediatric Dentistry 2021, 45, 48-53, doi:10.17796/1053-4625-45.1.9.

62. Sycinska-Dziarnowska, M.; Maglitto, M.; Woźniak, K.; Spagnuolo, G. Oral Health and Teledentistry Interest during the COVID-19 Pandemic. Journal of Clinical Medicine 2021, Vol. 10, Page 3532 2021, 10, 3532, doi:10.3390/JCM10163532.

63. Mandall, N.; O’Brien, K.; Brady, J.; Worthington, H.; Harvey, L. Teledentistry for Screening New Patient Orthodontic Referrals. Part 1: A Randomised Controlled Trial. British dental journal 2005, 199, 659-662, doi:10.1038/SJ.BDJ.4812930.

64. Giudice, A.; Barone, S.; Muraca, D.; Averta, F.; Diodati, F.; Antonelli, A.; Fortunato, L. Can Teledentistry Improve the Monitoring of Patients during the Covid-19 Dissemination? A Descriptive Pilot Study. International Journal of Environmental Research and Public Health 2020, 17, doi:10.3390/IJERPH17103399.

65. Maspero, C.; Abate, A.; Cavagnetto, D.; el Morsi, M.; Fama, A.; Farronato, M. Available Technologies, Applications and Benefits of Teleorthodontics. A Literature Review and Possible Applications during the COVID-19 Pandemic. Journal of Clinical Medicine 2020, Vol. 9, Page 1891 2020, 9, 1891, doi:10.3390/JCM9061891.

66. Deana, N.F.; Seiffert, A.; Aravena-rivas, Y.; Alonso-coello, P.; Muñoz-millán, P.; Espinoza-espinoza, G.; Pineda, P.; Zaror, C. Recommendations for Safe Dental Care: A Systematic Review of Clinical Practice Guidelines in the First Year of the COVID-19 Pandemic. International journal of environmental research and public health 2021, 18, doi:10.3390/IJERPH181910059.

67. Hoyte, T.; Kowlessar, A.; Mahabir, A.; Khemkaran, K.; Jagroo, P.; Jahoor, S. The Knowledge, Awareness, and Attitude Regarding COVID-19 among Trinidad and Tobago Dentists. A Cross-Sectional Survey. Oral 2021, Vol. 1, Pages 250-260 2021, 1, 250-260, doi:10.3390/ORAL1030024.

68. Estrich, C.G.; Mikkelsen, M.; Morrissey, R.; Geisinger, M.L.; Ioannidou, E.; Vujicic, M.; Araujo, M.W.B. Estimating COVID-19 Prevalence and Infection Control Practices among US Dentists. The Journal of the American Dental Association 2020, 151, 815-824, doi:10.1016/J.ADAJ.2020.09.005.

69. Gugnani, N.; Gugnani, S. Safety Protocols for Dental Practices in the COVID-19 Era. Evidence-Based Dentistry 2020 21:2 2020, 21, 56-57, doi:10.1038/s41432-020-0094-6.

70. SOUZA, A.F.; ARRUDA, J.A.A. de; COSTA, F.P.D.; BEMQUERER, L.M.; CASTRO, W.H.; CAMPOS, F.E.B.; KAKEHASI, F.M.; TRAVASSOS, D.V.; SILVA, T.A. Safety Protocols for Dental Care during the COVID-19 Pandemic: The Experience of a Brazilian Hospital Service. Brazilian Oral Research 2021, 35, doi:10.1590/18073107bor-2021.vol35.0070.

71. Bizzoca, M.E.; Campisi, G.; lo Muzio, L. An Innovative Risk-Scoring System of Dental Procedures and Safety Protocols in the COVID-19 Era. BMC Oral Health 2020, 20, 1-8, doi:10.1186/S12903-020-01301-5/TABLES/2.

72. Alsaegh, A.; Belova, E.; Vasil'ev, Y.; Zabroda, N.; Severova, L.; Timofeeva, M.; Dobrokhotov, D.; Leonova, A.; Mitrokhin, O. COVID-19 in Dental Settings: Novel Risk Assessment Approach. International Journal of Environmental Research and Public Health 2021, Vol. 18, Page 6093 2021, 18, 6093, doi:10.3390/IJERPH18116093.

73. Falahchai, M.; Babaee Hemmati, Y.; Hasanzade, M. Dental Care Management during the COVID-19 Outbreak. Special Care in Dentistry 2020, 40, 539-548, doi:10.1111/SCD.12523.

74. Attia, S.; Howaldt, H.P. Impact of COVID-19 on the Dental Community: Part I before Vaccine (BV). Journal of Clinical Medicine 2021, Vol. 10, Page 288 2021, 10, 288, doi:10.3390/JCM10020288.

75. Riad, A.; Pokorná, A.; Attia, S.; Klugarová, J.; Koščík, M.; Klugar, M. Prevalence of COVID-19 Vaccine Side Effects among Healthcare Workers in the Czech Republic. Journal of Clinical Medicine 2021, Vol. 10, Page 1428 2021, 10, 1428, doi:10.3390/JCM10071428. 
76. Chen, J.; Wang, R.; Gilby, N.B.; Wei, G.-W. Omicron (B.1.1.529): Infectivity, Vaccine Breakthrough, and Antibody Resistance. ArXiv 2021.

77. Cele, S.; Jackson, L.; Khan, K.; Khoury, D.; Moyo-Gwete, T.; Tegally, H.; Scheepers, C.; Amoako, D.; Karim, F.; Bernstein, M.; et al. SARS-CoV-2 Omicron Has Extensive but Incomplete Escape of Pfizer BNT162b2 Elicited Neutralization and Requires ACE2 for Infection. medRxiv 2021, 2021.12.08.21267417, doi:10.1101/2021.12.08.21267417.

78. Wilhelm, A.; Widera, M.; Grikscheit, K.; Toptan, T.; Schenk, B.; Pallas, C.; Metzler, M.; Kohmer, N.; Hoehl, S.; Helfritz, F.A.; et al. Reduced Neutralization of SARS-CoV-2 Omicron Variant by Vaccine Sera and Monoclonal Antibodies. medRxiv 2021, 2021.12.07.21267432, doi:10.1101/2021.12.07.21267432.

79. Gruell, H.; Vanshylla, K. MRNA Booster Immunization Elicits Potent Neutralizing Serum Activity against the SARS-CoV-2 Omicron Variant Available online: https://drive.google.com/file/d/13iHMR6rk3MKRFhDZmNuH3AAjR1uT8mEU/view (accessed on 15 December 2021).

80. HKUMed Finds Omicron SARS-CoV-2 Can Infect Faster and Better than Delta in Human Bronchus but with Less Severe Infection in Lung - News I HKUMed Available online: http://www.med.hku.hk/en/news/press/20211215-omicron-sars-cov-2-infection (accessed on 15 December 2021).

81. Cameroni, E.; Saliba, C.; Bowen, J.E.; Rosen, L.E.; Culap, K.; Pinto, D.; Marco, A. de; Zepeda, S.K.; Iulio, J. di; Zatta, F.; et al. Broadly Neutralizing Antibodies Overcome SARS-CoV-2 Omicron Antigenic Shift. bioRxiv 2021, 2021.12.12.472269, doi:10.1101/2021.12.12.472269.

82. Cele, S.; Jackson, L.; Khan, K.; Khoury, D.; Moyo-Gwete, T.; Tegally, H.; Scheepers, C.; Amoako, D.; Karim, F.; Bernstein, M.; et al. SARS-CoV-2 Omicron Has Extensive but Incomplete Escape of Pfizer BNT162b2 Elicited Neutralization and Requires ACE2 for Infection. medRxiv 2021, 2021.12.08.21267417, doi:10.1101/2021.12.08.21267417.

83. Upendran, A.; Gupta, R.; Geiger, Z. Dental Infection Control; 2021;

84. Enhancing Readiness for Omicron (B.1.1.529): Technical Brief and Priority Actions for Member States Available online: https://www.who.int/publications/m/item/enhancing-readiness-for-omicron-(b.1.1.529)-technical-briefand-priority-actions-for-member-states (accessed on 15 December 2021).

85. Classification of Omicron (B.1.1.529): SARS-CoV-2 Variant of Concern Available online: https://www.who.int/news/item/26-11-2021-classification-of-omicron-(b.1.1.529)-sars-cov-2-variant-of-concern (accessed on 12 December 2021).

86. Moderna - Moderna Announces Strategy to Address Omicron (B.1.1.529) SARS-CoV-2 Variant Available online: https:/investors.modernatx.com/news/news-details/2021/Moderna-Announces-Strategy-to-Address-OmicronB.1.1.529-SARS-CoV-2-Variant/default.aspx (accessed on 12 December 2021).

87. Karim, S.S.A.; Karim, Q.A. Omicron SARS-CoV-2 Variant: A New Chapter in the COVID-19 Pandemic. The Lancet 2021, 398, 2126-2128, doi:10.1016/S0140-6736(21)02758-6/ATTACHMENT/B8C47DC8-B485-4240-95DDFF7ADBB31E18/MMC1.PDF.

88. Pulliam, J.R.C.; Schalkwyk, C. van; Govender, N.; Gottberg, A. von; Cohen, C.; Groome, M.J.; Dushoff, J.; Mlisana, K.; Moultrie, H. Increased Risk of SARS-CoV-2 Reinfection Associated with Emergence of the Omicron Variant in South Africa. medRxiv 2021, 2021.11.11.21266068, doi:10.1101/2021.11.11.21266068.

89. WHO COVID-19 Weekly Epidemiological Update; 
90. Giudice, A.; Bennardo, F.; Antonelli, A.; Barone, S.; Fortunato, L. COVID-19 Is a New Challenge for Dental Practitioners: Advice on Patients' Management from Prevention of Cross Infections to Telemedicine. The Open Dentistry Journal 2020, 14, 298-304, doi:10.2174/1874210602014010298.

91. GISAID - HCov19 Variants Available online: https:/www.gisaid.org/hcov19-variants/ (accessed on 12 December 2021).

92. New Concerning Variant: B.1.1.529 - by Katelyn Jetelina Available online: https://yourlocalepidemiologist.substack.com/p/new-concerning-variant-b11529 (accessed on 12 December 2021).

93. SA Detects New COVID-19 Variant: Scientists I ENCA Available online: https://www.enca.com/news/southafrica-detects-new-covid-19-variant-scientists (accessed on 12 December 2021).

94. Baker, I.; Marzouqa, N.; Yaghi, B.N.; Adawi, S.O.; Yousef, S.; Sabooh, T.N.; Salhab, N.M.; Khrishi, H.M.; Qabaja, Y.; Riad, A.; et al. The Impact of Information Sources on COVID-19-Related Knowledge, Attitudes, and Practices (KAP) among University Students: A Nationwide Cross-Sectional Study. International Journal of Environmental Research and Public Health 2021, Vol. 18, Page 12462 2021, 18, 12462, doi:10.3390/IJERPH182312462.

95. van Kasteren, P.B.; van der Veer, B.; van den Brink, S.; Wijsman, L.; de Jonge, J.; van den Brandt, A.; Molenkamp, R.; Reusken, C.B.E.M.; Meijer, A. Comparison of Seven Commercial RT-PCR Diagnostic Kits for COVID-19. Journal of Clinical Virology 2020, 128, 104412, doi:10.1016/j.jcv.2020.104412.

96. Mushtaq, M.Z.; Shakoor, S.; Kanji, A.; Shaheen, N.; Nasir, A.; Ansar, Z.; Ahmed, I.; Mahmood, S.F.; Hasan, R.; Hasan, Z. Discrepancy between PCR Based SARS-CoV-2 Tests Suggests the Need to Re-Evaluate Diagnostic Assays. BMC Research Notes 2021, 14, 316, doi:10.1186/s13104-021-05722-5.

97. Planas, D.; Saunders, N.; Maes, P.; Guivel-Benhassine, F.; Planchais, C.; Buchrieser, J.; Bolland, W.-H.; Porrot, F.; Staropoli, I.; Lemoine, F.; et al. Considerable Escape of SARS-CoV-2 Variant Omicron to Antibody Neutralization. bioRxiv 2021, 2021.12.14.472630, doi:10.1101/2021.12.14.472630.

98. Brandal, L.T.; MacDonald, E.; Veneti, L.; Ravlo, T.; Lange, H.; Naseer, U.; Feruglio, S.; Bragstad, K.; Hungnes, O.; Ødeskaug, L.E.; et al. Outbreak Caused by the SARS-CoV-2 Omicron Variant in Norway, November to December 2021. Euro surveillance : bulletin Europeen sur les maladies transmissibles = European communicable disease bulletin 2021, 26, 2101147, doi:10.2807/1560-7917.ES.2021.26.50.2101147/CITE/PLAINTEXT.

99. Kuhlmann, C.; Mayer, C.K.; Claassen, M.; Maponga, T.G.; Sutherland, A.D.; Suliman, T.; Shaw, M.; Preiser, W. Breakthrough Infections with SARS-CoV-2 Omicron Variant Despite Booster Dose of MRNA Vaccine. SSRN Electronic Journal 2021, doi:10.2139/SSRN.3981711.

100. Martinez-Sobrido, L.; Almazan Toral, F.; Faraz Ahmed, S.; Abdul Quadeer, A.; McKay, M.R. SARS-CoV-2 T Cell Responses Elicited by COVID-19 Vaccines or Infection Are Expected to Remain Robust against Omicron. Viruses 2022, Vol. 14, Page 79 2022, 14, 79, doi:10.3390/V14010079.

101. Ahmed, S.F.; Quadeer, A.A.; McKay, M.R. SARS-CoV-2 T Cell Responses Elicited by COVID-19 Vaccines or Infection Are Expected to Remain Robust against Omicron. Viruses 2022, 14, doi:10.3390/v14010079.

102. SARS-CoV-2 Variants of Concern as of 13 January 2022 Available online: https://www.ecdc.europa.eu/en/covid19/variants-concern (accessed on 14 January 2022).

103. Wolter, N.; Jassat, W.; Walaza, S.; Welch, R.; Moultrie, H.; Groome, M.; Amoako, D.G.; Everatt, J.; Bhiman, J.N.; Scheepers, C.; et al. Early Assessment of the Clinical Severity of the SARS-CoV-2 Omicron Variant in South Africa. medRxiv 2021, 2021.12.21.21268116, doi:10.1101/2021.12.21.21268116. 
104. Keeton, R.; Tincho, M.B.; Ngomti, A.; Baguma, R.; Benede, N.; Suzuki, A.; Khan, K.; Cele, S.; Bernstein, M.; Karim, F.; et al. SARS-CoV-2 Spike T Cell Responses Induced upon Vaccination or Infection Remain Robust against Omicron. medRxiv 2021, 2021.12.26.21268380, doi:10.1101/2021.12.26.21268380.

105. Dolgin, E. Omicron Is Supercharging the COVID Vaccine Booster Debate. Nature 2021, doi:10.1038/D41586-02103592-2.

106. Volgenant, C.M.C.; de Soet, J.J. Cross-Transmission in the Dental Office: Does This Make You Ill? Current Oral Health Reports 2018, 5, 221, doi:10.1007/S40496-018-0201-3.

107. Benzian, H.; Beltrán-Aguilar, E.; Niederman, R. Systemic Management of Pandemic Risks in Dental Practice: A Consolidated Framework for COVID-19 Control in Dentistry. Frontiers in Medicine 2021, 8, 196, doi:10.3389/FMED.2021.644515/BIBTEX.

108. Nemati, S. Impacts of COVID-19 Outbreak on Dentistry Dimensions. Iranian Journal of Medical Sciences 2021, 46, 149, doi:10.30476/IJMS.2021.47491.

109. Higgins, V.; Sohaei, D.; Diamandis, E.P.; Prassas, I. COVID-19: From an Acute to Chronic Disease? Potential Long-Term Health Consequences. Critical Reviews in Clinical Laboratory Sciences 2021, 58, 297-310, doi:10.1080/10408363.2020.1860895.

110. Salehi, S.; Reddy, S.; Gholamrezanezhad, A. Long-Term Pulmonary Consequences of Coronavirus Disease 2019 (COVID-19): What We Know and What to Expect. Journal of Thoracic Imaging 2020, 35, W87-W89, doi:10.1097/RTI.0000000000000534.

111. Shaw, B.; Daskareh, M.; Gholamrezanezhad, A. The Lingering Manifestations of COVID-19 during and after Convalescence: Update on Long-Term Pulmonary Consequences of Coronavirus Disease 2019 (COVID-19). Radiologia Medica 2021, 126, 40-46, doi:10.1007/s11547-020-01295-8.

112. McDonald, L.T. Healing after COVID-19: Are Survivors at Risk for Pulmonary Fibrosis? American Journal of Physiology - Lung Cellular and Molecular Physiology 2021, 320, L257-L265, doi:10.1152/AJPLUNG.00238.2020.

113. Mishra, S.K.; Tripathi, T. One Year Update on the COVID-19 Pandemic: Where Are We Now? Acta Tropica 2021, 214, 105778, doi:10.1016/j.actatropica.2020.105778.

114. Baig, A.M. Deleterious Outcomes in Long-Hauler COVID-19: The Effects of SARS-CoV-2 on the CNS in Chronic COVID Syndrome. ACS Chemical Neuroscience 2020, 11, 4017-4020, doi:10.1021/acschemneuro.0c00725.

115. Valenzano, A.; Scarinci, A.; Monda, V.; Sessa, F.; Messina, A.; Monda, M.; Precenzano, F.; Mollica, M.P.; Carotenuto, M.; Messina, G.; et al. The Social Brain and Emotional Contagion: Covid-19 Effects. Medicina (Lithuania) 2020, 56, 1-10, doi:10.3390/medicina56120640.

116. Evans, P.C.; Rainger, G.E.; Mason, J.C.; Guzik, T.J.; Osto, E.; Stamataki, Z.; Neil, D.; Hoefer, I.E.; Fragiadaki, M.; Waltenberger, J.; et al. Endothelial Dysfunction in COVID-19: A Position Paper of the ESC Working Group for Atherosclerosis and Vascular Biology, and the ESC Council of Basic Cardiovascular Science. Cardiovascular Research 2020, 116, 2177-2184, doi:10.1093/cvr/cvaa230.

117. Madjunkov, M.; Dviri, M.; Librach, C. A Comprehensive Review of the Impact of COVID-19 on Human Reproductive Biology, Assisted Reproduction Care and Pregnancy: A Canadian Perspective. Journal of Ovarian Research 2020, 13, 1-18, doi:10.1186/s13048-020-00737-1.

118. Wang, N.; Qin, L.; Ma, L.; Yan, H. Effect of Severe Acute Respiratory Syndrome Coronavirus-2 (SARS-CoV-2) on Reproductive System. Stem Cell Research 2021, 52, 102189, doi:10.1016/j.scr.2021.102189.

119. ZostaňZdravý - Aplikácia Available online: https://www.zostanzdravy.sk/en (accessed on 14 December 2021).

120. Home - DentalMonitoring Available online: https://dental-monitoring.com/ (accessed on 15 December 2021). 
121. Thurzo, A.; Urbanová, W.; Novák, B.; Waczulíková, I.; Varga, I. 3D Printed Orthodontic Distalizer with Individual Base for Tooth-Borne Hybrid Approach in Class II Unilateral Malocclusions Treatment. 2021, doi:10.20944/PREPRINTS202111.0547.V1.

122. Thurzo, A.; Kočiš, F.; Novák, B.; Czako, L.; Varga, I. Three-Dimensional Modeling and 3D Printing of Biocompatible Orthodontic Power-Arm Design with Clinical Application. Applied Sciences 2021, 11, 9693, doi:10.3390/app11209693.

123. accessed on 14th December 2021 COVID ENG Available online: https://sangreazul.typeform.com/BioSafetyENG (accessed on 14 December 2021).

124. WHO Director-General's Opening Remarks at the Media Briefing on COVID-19 - 14 December 2021 Available online: https://www.who.int/director-general/speeches/detail/who-director-general-s-opening-remarks-at-themedia-briefing-on-covid-19---14-december-2021 (accessed on 16 December 2021).

125. Bjornevik, K.; Cortese, M.; Healy, B.C.; Kuhle, J.; Mina, M.J.; Leng, Y.; Elledge, S.J.; Niebuhr, D.W.; Scher, A.I.; Munger, K.L.; et al. Longitudinal Analysis Reveals High Prevalence of Epstein-Barr Virus Associated with Multiple Sclerosis. Science (New $\quad$ York, doi:10.1126/SCIENCE.ABJ8222/SUPPL_FILE/SCIENCE.ABJ8222_DATA_S1.PDF.

126. Wang, L.; Berger, N.A.; Kaelber, D.C.; Davis, P.B.; Volkow, N.D.; Xu, R. Comparison of Outcomes from COVID Infection in Pediatric and Adult Patients before and after the Emergence of Omicron. medRxiv 2022, 2021.12.30.21268495, doi:10.1101/2021.12.30.21268495. 